\title{
Proof Theory
}

A selection of papers

from the Leeds Proof Theory Programme 1990

edited by

Peter Aczel

University of Manchester

Harold Simmons

University of Manchester

and

Stanley S. Wainer

University of Leeds 
Published by the Press Syndicate of the University of Cambridge The Pitt Building, Trumpington Street, Cambridge CB2 1RP

40 West 20th Street, New York, NY 10011-4211, USA

10 Stamford Road, Oakleigh, Victoria 3166, Australia

(c) Cambridge University Press 1992

First published 1992

Printed in the Great Britain at the University Press, Cambridge

British Library cataloguing in publication data available

Library of Congress cataloguing in publication data available

ISBN $052141413 \mathrm{X}$ hardcover 


\section{CONTENTS}

Preface vii

Programme of lectures $\quad$ ix

S. WAINER and L. WALLEN

Basic proof theory 1

W. POHLERS

A short course in ordinal analysis $\quad 27$

H. SCHWICHTENBERG

$\begin{array}{ll}\text { Proofs as programs } & 79\end{array}$

W. BUCHHOLZ

A simplified version of local predicativity $\quad 115$

S. BUSS

A note on bootstrapping intuitionistic bounded arithmetic $\quad 149$

E. CICHON

$\begin{array}{ll}\text { Termination orderings and complexity characterisations } & 171\end{array}$

S. FEFERMAN

Logics for termination and correctness of functional programs, II.

Logics of strength $P R A$

D. HOWE

Reflecting the semantics of reflected proof

M. RATHJEN

Fragments of Kripke-Platek set theory with infinity

J. TUCKER and J. ZUCKER

Provable computable selection functions on abstract structures 


\title{
A simplified version of local predicativity
}

\author{
W. BUCHHOLZ
}

Reproduced from 'Proof Theory' edited by Aczell, Simmons \& Wainer.

(C) 1993 Cambridge University Press 



\title{
A Simplified Version of Local Predicativity
}

\author{
W. BUCHHOLZ
}

Mathematisches Institut der Universität München ${ }^{1}$

The method of local predicativity as developed by Pohlers in [10],[11],[12] and extended to subsystems of set theory by Jäger in [4],[5],[6] is a very powerful tool for the ordinal analysis of strong impredicative theories. But up to now it suffers considerably from the fact that it is based on a large amount of very special ordinal theoretic prerequisites. This is true even for the most recent (very polished) presentation of local predicativity in (Pohlers [15]). The purpose of the present paper is to expose a simplified and conceptually improved version of local predicativity which - besides some very elementary facts on ordinal addition, multiplication, and exponentiation - requires only amazingly little ordinal theory. (All necessary nonelementary ordinal theoretic prerequisites can be developed from scratch on just two pages, as we will show in section 4.) The most important feature of our new approach however seems to be its conceptual clarity and flexibility, and in particular the fact that its basic concepts (i.e. the infinitary system $\mathrm{RS}^{\infty}$ and the notion of an $\mathcal{H}$-controlled $\mathrm{RS}^{\infty}$-derivation) are in no way related to any system of ordinal notations or collapsing functions. Our intention with this paper is to make the fascinating field of 'admissible proof theory' (created by Jäger and Pohlers) more easily accessible for non-prooftheorists, and to provide a technically and conceptually well developed basis for further research in this area. We think a good way to accomplish this goal is to apply our method to one particularly interesting (and strong) theory, namely the system KPi first analyzed by Jäger and Pohlers in [9], and to carry out the ordinal analysis for this theory in full detail. Accordingly the whole paper is devoted to the proof of the following

\footnotetext{
${ }^{1}$ The final version of this paper was written while the author was visiting Carnegie Mellon University during the academic year 1990/91. I would like to express my sincere thanks to Wilfried Sieg (who invited me) and all members of the Philosophy Department of CMU for their generous hospitality.
} 


\section{MAIN THEOREM}

If $\phi$ is a $\Sigma_{1}$-sentence (in the language $\mathcal{L}$ of set theory) such that $\mathrm{KPi}$ proves $\forall x\left(\operatorname{Ad}(x) \rightarrow \phi^{x}\right)$ then $L_{v} \vDash \phi$, where $v:=\psi_{\Omega_{1}}\left(\varepsilon_{\mathrm{I}+1}\right)$.

We assume that the reader has some familiarity with Kripke-Platek set theory and with theories for iterated admissible sets like KPi. Therefore here we only add two short remarks concerning the significance of the above theorem. For extensive background information we refer the reader to $[6],[8],[13],[15],[16]$.

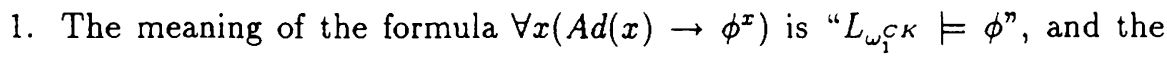
ordinal $v$ is in fact less than $\omega_{1}^{C K}$. So the theorem gives a specific ordinal $v<\omega_{1}^{C K}$ such that $L_{v}$ is a model of each $\Sigma_{1}$-sentences $\phi$ for which KPi proves that $L_{\omega_{1}^{C K}}$ is a model of $\phi$.

2. As shown in (Rathjen [19]) the above theorem implies that $v$ is an upper bound for $|\mathrm{KPi}|$, the proof-theoretic ordinal of KPi defined by

$|\mathrm{KPi}|:=\sup \{|\prec|: \prec$ prim.rec.wellord. with $\mathrm{KPi} \vdash$ “ $\prec$ is wellfounded" $\}$,

where $|\prec|$ denotes the ordertype of $\prec$.

(For the readers convenience we repeat the proof given in [19]. Suppose that $\prec$ is a primitive recursive wellordering of $\omega$ such that $\mathrm{KPi} \vdash$ " $\prec$ is wellfounded", and let $\phi_{\prec}$ be the $\Sigma_{1}$-sentence expressing that there is a function $f: \omega \rightarrow$ On with $f(n)=\{f(m): m \prec n\}(\forall n \in \omega)$. Then by [8](Theorem 4.6) we have KPiF $\forall x\left(\operatorname{Ad}(x) \rightarrow \phi_{\prec}^{x}\right)$, and the Main Theorem yields $L_{v} \vDash \phi_{\prec}$, i.e. there exists a function $f \in L_{v}$ with $\operatorname{dom}(f)=\omega$ and $f(n)=\{f(m): m \prec n\}(\forall n \in \omega)$. But this implies $|\prec|=\operatorname{ran}(f) \in L_{v}$, i.e. $|\prec|<v$.)

\section{Remark}

The method introduced in this paper can also be used to simplify considerably Rathjen's [19] ordinal analysis of KPM, a theory much stronger than $\mathrm{KPi}$. This will be carried out in a forthcoming paper [3]. For the sake of completeness we want to mention that another ordinal analysis of KPM has been obtained independently by T. Arai [1]. 


\section{The language $\mathcal{L}_{R S}$ of ramified set theory}

Let $\mathcal{L}$ denote the usual first order language of set theory whose only nonlogical symbol is the binary predicate constant $\epsilon$. The language $\mathcal{L}_{\text {Ad }}$ is obtained from $\mathcal{L}$ by adding the unary predicate constant $A d$. The language $\mathcal{L}_{R S}$ of ramified set theory is obtained from $\mathcal{L}_{\text {Ad }}$ by adding a certain class $\mathcal{T}$ of individual constants, the so-called set terms or RS-terms. The definition of $\mathcal{T}$ will be given below. Before that we introduce some technical notions and abbreviations. In this context we use the letters $u, v$ to denote both, individual variables and RS-terms. Individual variables are indicated by $w, x, y, z$.

The atomic formulas of $\mathcal{L}_{R S}$ are $u \in v, \neg(u \in v), A d(u), \neg A d(u)$. The formulas of $\mathcal{L}_{R S}$ are built up from atomic formulas by means of $\wedge, \vee, \forall, \exists$. The negation $\neg A$ of an $\mathcal{L}_{R S}$-formula $A$ is defined via de Morgan's laws. A quantifier (occurrence) $\forall x[\exists x]$ in a formula $A$ is called restricted (or bounded) if its range (i.e. the subformula following that quantifier) is of the form $x \in v \rightarrow B(x)[x \in v \wedge B(x)]$ with $x \not \equiv v$. A formula $A$ is called a $\Delta_{0}$-formula if it contains no unrestricted quantifier. The $\Delta_{0}$-formulas of the language $\mathcal{L}_{R S}$ are called $R S$-formulas. As usual the formula obtained from $A$ by restricting every unrestricted quantifier to $u$ is denoted by $A^{u}$.

From now on we use $A, B, C$ to denote $R S$-sentences (i.e. closed RS-formulas), and $A\left(x_{1}, \ldots, x_{n}\right)$, etc. to denote RS-formulas which have all their free variables among $x_{1}, \ldots, x_{n}$. Correspondingly we use $\phi, \psi, \phi\left(x_{1}, \ldots, x_{n}\right)$, etc. to denote sentences and formulas of the language $\mathcal{L}_{\text {Add }}$. Finite sequences of variables are abbreviated by $\vec{x}, \vec{y}, \ldots$.

\section{Abbreviations}

$$
\begin{aligned}
& A(\vec{x}) \rightarrow B(\vec{x}): \equiv \neg A(\vec{x}) \vee B(\vec{x}) \\
& \forall x \in v B(x, \vec{y}): \equiv \forall x(x \in v \rightarrow B(x, \vec{y})) \quad(x \not \equiv v) \\
& \exists x \in v B(x, \vec{y}): \equiv \exists x(x \in v \wedge B(x, \vec{y}) \quad(x \not \equiv v) \\
& u \subseteq v: \equiv \forall x \in u(x \in v) \\
& u=v: \equiv u \subseteq v \wedge v \subseteq u \\
& u \notin v: \equiv \neg(u \in v) \\
& u \neq v: \equiv \neg(u=v) \\
& \operatorname{tran}(u): \equiv \forall x \in u \forall y \in x(y \in u) \\
& \text { infinite }(u): \equiv \exists x \in u(x \subseteq x) \wedge \forall x \in u \exists y \in u(x \in y)
\end{aligned}
$$


Definition 1.1 (RS-terms and their levels)

1. For every ordinal $\alpha$ the constant $L_{\alpha}$ is an RS-term of level $\alpha$.

2. If $\phi\left(x, y_{1}, \ldots, y_{n}\right)$ is an $\mathcal{L}_{A d}$-formula which contains at least one free occurrence of $x$, and if $a_{1}, \ldots, a_{n}$ are RS-terms of levels $<\alpha$ (where $\alpha>0)$, then

$$
\left[x \in \mathrm{L}_{\alpha}: \phi^{\mathrm{L}_{\alpha}}\left(x, a_{1}, \ldots, a_{n}\right)\right]
$$

is an RS-term of level $\alpha$.

We denote the class of all RS-terms by $\mathcal{T}$, and the class of all RS-terms of level less than $\alpha$ by $\mathcal{T}_{\alpha}$.

In the following RS-terms are denoted by the letters $a, b, c, s, t$.

Note that all variables occurring in an RS-term are bound.

Definition 1.2 (Definition of $k(\theta)$ and $|\theta|$ )

If $\theta$ is an RS-term or RS-formula we set

$\mathrm{k}(\theta):=\left\{\alpha \in \mathrm{On}: \mathrm{L}_{\alpha}\right.$ occurs in $\left.\theta\right\} \quad$ and $\quad|\theta|:=\max (\mathrm{k}(\theta) \cup\{0\})$

Here all occurrences of $L_{\alpha}$, i.e. also those inside of subterms of $\theta$ are counted. (Example: $k\left(\left[x \in L_{\alpha}: L_{\beta} \in x\right] \in L_{\gamma}\right)=\{\alpha, \beta, \gamma\}$.)

For technical reasons we also define $\mathrm{k}(0):=\mathrm{k}(1):=\emptyset, \quad|0|:=|1|:=0$.

\section{Remark}

For each $t \in \mathcal{T}$ we have level of $t=|t| \in \mathrm{k}(t)$.

Hence $\mathcal{T}_{\alpha}=\{t \in \mathcal{T}:|t|<\alpha\}$.

\section{Definition 1.3}

For RS-terms $a, b$ with $|a|<|b|$ we set

$a \stackrel{\circ}{\epsilon} b: \equiv\left\{\begin{array}{ll}B(a) & \text { if } b \equiv\left[x \in L_{B}: B(x)\right] \\ a \notin L_{0} & \text { if } b \equiv L_{B}\end{array} \quad\right.$ and $\quad a \stackrel{\circ}{\notin} b: \equiv \neg(a \stackrel{\circ}{\epsilon} b)$.

We now are going to intoduce a semantics for the language $\mathcal{L}_{R S}$. For this we fix some class $R$ of ordinals which will then be used for defining the meaning of the predicate constant $A d$. The intended interpretation of $A d$ is the class $\left\{L_{\kappa}: \omega<\kappa\right.$ admissible $\}$. Therefore we should take $R$ as the class $\{\kappa: \omega<\kappa$ admissible $\}$. But for the purpose of this paper it is much more convenient to define $\mathrm{R}$ as a class of uncountable regular cardinals as we wiil do in section 4. For the meantime it suffices to make the following 
Assumption: $\mathrm{R}$ is a nonempty class of $\varepsilon$-numbers.

In the following the letters $\kappa, \pi, \tau$ always denote elements of $\mathrm{R}$.

Definition 1.4 (Semantics of $\mathcal{L}_{R S}$ )

By recursion on $|a|$ we define, for each $a \in \mathcal{T}$, a set $\mathrm{s}(a)$ as follows:

1. $\mathrm{s}\left(\mathrm{L}_{\alpha}\right):=\mathrm{s}\left[\mathcal{T}_{\alpha}\right]:=\left\{\mathrm{s}(t): t \in \mathcal{T}_{\alpha}\right\}$

2. $s\left(\left[x \in \mathrm{L}_{\alpha}: \phi^{\mathrm{L}_{\alpha}}\left(x, a_{1}, \ldots, a_{n}\right)\right]\right):=$ $\left\{\mathrm{s}(t): t \in \mathcal{T}_{\alpha} \&\left(\mathrm{~s}\left(\mathrm{~L}_{\alpha}\right), \in, \underline{A d_{\alpha}}\right) \models \phi\left(s(t), \mathbf{s}\left(a_{1}\right), \ldots, \mathrm{s}\left(a_{n}\right)\right)\right\}$, where $\underline{A d}_{\alpha}:=\left\{\mathbf{s}\left(\mathrm{L}_{\kappa}\right): \kappa<\alpha\right\}$, and $\in$ is the standard membership relation.

Now let $\mathcal{M}$ be the first order structure for $\mathcal{L}_{R S}$ consisting of

- the universe $\mathbf{s}[\mathcal{T}]:=\{\mathbf{s}(t): t \in \mathcal{T}\}$,

- the membership relation $\epsilon$,

- the class $\underline{A d}:=\left\{\mathbf{s}\left(\mathrm{L}_{\kappa}\right): \kappa \in \mathrm{R}\right\}$,

- the family $(s(a))_{a \in \mathcal{T}}$.

Then for each $\mathcal{L}_{R S}$-sentence $\Phi$ we set: $\quad \models \Phi: \Longleftrightarrow \mathcal{M} \models \Phi$.

Obviously $\mathbf{s}[\mathcal{T}]$ as well as all $\mathbf{s}\left(\mathrm{L}_{\alpha}\right)(\alpha \in \mathrm{On})$ are transitive, and one easily verifies the following equivalences:

$$
\begin{aligned}
& (\vDash 1) \quad \vDash A d(a) \Longleftrightarrow \exists \kappa \leq|a|\left(\vDash L_{\kappa}=a\right) \\
& (\vDash 2) \quad \vDash a \in b \Longleftrightarrow \exists t \in \mathcal{T}_{|b|}(\vDash t \stackrel{\circ}{\in} b \wedge t=a) \\
& (\vDash 3) \quad \models \exists x \in b A(x) \Longleftrightarrow \exists t \in \mathcal{T}_{|b|}(\models t \stackrel{\circ}{\epsilon} b \wedge A(t))
\end{aligned}
$$

(For the proof of $(\models 1)$ one has to use the fact that $|a|<\kappa$ implies $s(a) \in \mathbf{s}\left(L_{\kappa}\right)$ and thus $\mathrm{s}(a) \neq \mathrm{s}\left(\mathrm{L}_{\kappa}\right)$.)

\section{Lemma 1.5}

Let $\left(L_{\alpha}\right)_{\alpha \in O \text { n }}$ be the constructible hierarchy.

Then for each $\mathcal{L}$-sentence $o$ and each $3 \leq \min (R)$ we have

$\models 0^{L_{\beta}} \Longleftrightarrow L_{3} \vDash 0$.

Proof.

For $\beta \leq \min (R) \quad$ let $\mathcal{T}_{3}^{-}:=\left\{t \in \mathcal{T}_{\beta}:\right.$ Ad does not occur in $\left.t\right\}$. Using $\forall \beta \leq \min (R)\left(\underline{A d}_{\beta}=\emptyset\right)$ we obtain $s\left[\mathcal{T}_{\beta}\right]=s\left[\mathcal{T}_{\beta}^{-}\right]$, for all $\beta \leq \min (R)$. Now 
by induction on $\beta$ it follows that $L_{\beta}=s\left[\mathcal{T}_{\beta}\right]=s\left(L_{\beta}\right)$. Hence $\vDash \phi^{L_{\beta}} \Leftrightarrow s[\mathcal{T}] \vDash \phi^{L_{\beta}} \Leftrightarrow s\left(L_{\beta}\right) \models \phi \Leftrightarrow L_{\beta} \vDash \phi$.

The next definition is motivated by $(\models 1)-(\vDash 3)$.

\section{Definition $1.6^{2}$}

To each RS-sentence $A$ we assign a certain (infinitary) conjunction $\wedge\left(A_{\iota}\right)_{\iota} \in J$ or disjunction $\mathrm{V}\left(A_{\iota}\right)_{\iota \in J}$ of RS-sentences and we indicate this assignment by writing $A \simeq \wedge\left(A_{\iota}\right)_{\iota \in J}, A \simeq \bigvee\left(A_{\iota}\right)_{\iota \in J}$, resp.

1. $A d(a): \simeq \bigvee(t=a)_{t \in J}$ with $J:=\left\{\mathrm{L}_{\kappa}: \kappa \in \mathrm{R} \& \kappa \leq|a|\right\}$

2. $a \in b: \simeq \bigvee(t \stackrel{\circ}{\epsilon} b \wedge t=a)_{t \in J}$ with $J:=\mathcal{T}_{|b|}$

3. $\exists x \in b A(x): \simeq \bigvee(t \stackrel{\circ}{\in} b \wedge A(t))_{t \in J}$ with $J:=\mathcal{T}_{|b|}$

4. $\left(A_{0} \vee A_{1}\right): \simeq \bigvee\left(A_{\iota}\right)_{\iota \in\{0,1\}}$

5. $\neg A: \simeq \wedge\left(\neg A_{\iota}\right)_{\iota \in J}$, if $\mathrm{A}$ is one of the formulas under 1.-4.

As an immediate consequence of $(\models 1-\vDash 3)$ we obtain the following lemma.

\section{Lemma 1.7}

(i) $\vDash \wedge\left(A_{\imath}\right)_{\iota \in J} \Longleftrightarrow \forall \iota \in J\left(\vDash A_{\iota}\right)$

(ii) $\models \vee\left(A_{\iota}\right)_{\iota \in J} \Longleftrightarrow \exists \iota \in J\left(\vDash A_{\iota}\right)$

In the formulation of the above lemma we have already used the following notational convention to which we stick through the whole paper.

\section{Notational convention}

By writing $\wedge\left(A_{\iota}\right)_{\iota \in J}\left[\bigvee\left(A_{\iota}\right)_{\iota \in J}\right.$, resp.] we indicate a certain $R S$-sentence $A$ such that $A \simeq \wedge\left(A_{\iota}\right)_{\iota \in J}\left[A \simeq \bigvee\left(A_{\iota}\right)_{\iota \in J}\right.$, resp. $]$.

We now define a rank-function for RS-sentences in such a way that $\forall \iota \in J\left(\operatorname{rk}\left(A_{\iota}\right)<\operatorname{rk}(A)\right)$ whenever $A \simeq \hat{V}\left(A_{\iota}\right)_{\iota \in J}$.

Definition 1.8 (the rank of RS-sentences and RS-terms)

The $\operatorname{rank} \operatorname{rk}(\theta)$ of an RS-sentence or RS-term $\theta$ is defined by induction on the

\footnotetext{
${ }^{2}$ this elegant way of turning a formal language into a fragment of infinitary propositional logic I have first seen in an unpublished manuscript by W.W.Tait
} 
number of symbols occurring in $\theta$ as follows:

1. $\operatorname{rk}\left(\mathrm{L}_{\alpha}\right):=\omega \cdot \alpha$

2. $\operatorname{rk}\left(\left[x \in \mathrm{L}_{\alpha}: A(x)\right]\right):=\max \left\{\omega \cdot \alpha+1, \operatorname{rk}\left(A\left(\mathrm{~L}_{0}\right)\right)+2\right\}$

3. $\operatorname{rk}(A d(a)):=\operatorname{rk}(\neg A d(a)):=\operatorname{rk}(a)+5$

4. $\operatorname{rk}(a \in b):=\operatorname{rk}(a \notin b):=\max \{\operatorname{rk}(a)+6, \operatorname{rk}(b)+1\}$

5. $\operatorname{rk}(\exists x \in b A(x)):=\operatorname{rk}(\forall x \in b A(x)):=\max \left\{\operatorname{rk}(b), \operatorname{rk}\left(A\left(L_{0}\right)\right)+2\right\}$

6. $\operatorname{rk}(A \wedge B):=\operatorname{rk}(A \vee B):=\max \{\operatorname{rk}(A), \operatorname{rk}(B)\}+1$

\section{Lemma 1.9}

Let $A \simeq \bigvee\left(A_{\iota}\right)_{\iota \in J}$ or $A \simeq \wedge\left(A_{\iota}\right)_{\iota \in J}$. Then the following holds.
a) $\operatorname{rk}(A)=\omega \cdot|A|+n$, for some $n \in \omega$
b) $\operatorname{rk}\left(A_{\iota}\right)<\operatorname{rk}(A)$, for all $\iota \in J$
c) $\mathrm{k}(\iota) \subseteq \mathrm{k}\left(A_{\iota}\right) \subseteq \mathrm{k}(A) \cup \mathrm{k}(\iota)$, for all $\iota \in J$.
d) $\operatorname{rk}(A)=\omega \cdot \alpha \Longrightarrow A \equiv \exists x \in \mathrm{L}_{\alpha} B(x)$ or $A \equiv \forall x \in \mathrm{L}_{\alpha} B(x)$
e) $\operatorname{rk}(A)=\operatorname{rk}(\neg A)$

Proof.

The easy proofs of a),c),d),e) are left to the reader. The proof of b) is obtained by successively verifying the following propositions.

(1) $|c|<\left|A\left(\mathrm{~L}_{0}\right)\right| \Longrightarrow \operatorname{rk}(A(c))=\operatorname{rk}\left(A\left(\mathrm{~L}_{0}\right)\right)$

(2) $|c|<\beta \Longrightarrow \operatorname{rk}(A(c))<\max \left\{\omega \beta, \operatorname{rk}\left(A\left(\mathrm{~L}_{0}\right)\right)+1\right\}$

(3) $|c|<|b| \Longrightarrow \operatorname{rk}(c \stackrel{\circ}{\in} b)+1<\operatorname{rk}(b)$

(4) $\operatorname{rk}\left(L_{0} \in L_{0}\right)=6$ and $\operatorname{rk}\left(L_{0} \in b\right)=\operatorname{rk}(b)+1$ for $b \not \equiv L_{0}$

(5) $\mathrm{rk}\left(\mathrm{L}_{0}=\mathrm{L}_{0}\right)=9$ and $\operatorname{rk}(a=b)=\max \{\operatorname{rk}(a), \operatorname{rk}(b)\}+4$, if $a \not \equiv \mathrm{L}_{0}$ or $b \not \equiv \mathrm{L}_{0}$

(6) $|c|<|b| \Longrightarrow \operatorname{rk}(c \stackrel{\circ}{\in} b \wedge c=a)<\operatorname{rk}(a \in b)$ and $\operatorname{rk}(c \stackrel{\circ}{\in} b \wedge A(c))<\operatorname{rk}(\exists x \in b A(x))$

(7) $\kappa \leq|a| \Longrightarrow \operatorname{rk}\left(\mathrm{L}_{\kappa}=a\right)=\operatorname{rk}(a)+4$

We close this section by some additional definitions and abbreviations.

\section{Definition 1.10}

1. A formula which contains no unrestricted universal quantifier is called a $\Sigma_{1}$-formula.

2. The set of all RS-sentences $A \equiv \phi^{L_{\kappa}}(\vec{a})$ with $\phi(\vec{x}) \in \Sigma_{1}$ and $\vec{a} \in \mathcal{T}_{\kappa}$ is denoted by $\Sigma(\kappa)$. 
3. For $A \equiv \phi^{L_{\kappa}}(\vec{a}) \in \Sigma(\kappa)$ we set $A^{(u, \kappa)}: \equiv \phi^{u}(\vec{a})$, and we abbreviate $A^{L_{\beta}}$ by $A^{\beta}$, and $A^{\left(\mathrm{L}_{\beta}, \kappa\right)}$ by $A^{(\beta, \kappa)}$.

\section{Definition 1.11}

1. $\mathcal{T}^{0,1}:=\mathcal{T} \cup\{0,1\}$ and $J \mid \alpha:=\{\iota \in J:|\iota|<\alpha\}$ for $J \subseteq \mathcal{T}^{0,1}$.

2. We use $\Theta$ to denote finite sequences consisting of RS-sentences and elements of $\mathcal{T}^{0,1}$, and for $\Theta=\left(\theta_{1}, \ldots, \theta_{n}\right)$ we set $k(\Theta):=\mathrm{k}\left(\theta_{1}\right) \cup \ldots \cup \mathrm{k}\left(\theta_{n}\right)$.

3. Finite sequences of RS-sentences are called $R S$-sequents and indicated by the letters $\Gamma, \Gamma^{\prime} . \quad$ For $\Gamma=\left(A_{0}, \ldots, A_{n}\right)$ we set $\quad \models \Gamma: \Longleftrightarrow \models A_{0} \vee \ldots \vee A_{n}$.

\section{Definition 1.12}

For each ordinal $\alpha$ we set $\quad \alpha^{\mathrm{R}}:= \begin{cases}\min \{\kappa \in \mathrm{R}: \alpha<\kappa\} & \text { if } \exists \kappa \in \mathrm{R}(\alpha<\kappa) \\ \alpha & \text { otherwise }\end{cases}$

The letters $\alpha, \beta, \gamma, \delta, \mu, \sigma, \xi, \eta, \zeta$ always denote ordinals. On denotes the class of all ordinals, Lim the class of all limit numbers, and $\mathcal{P}(\mathrm{On})$ the class of all subsets of On. Every ordinal $\alpha$ is identified with the set $\{\xi \in \mathrm{On}: \xi<\alpha\}$ of its predecessors. For $\alpha \leq \beta$ we set $[\alpha, \beta]:=\{\xi: \alpha \leq \xi \leq \beta\}$ and $[\alpha, \beta[:=\{\xi$ : $\alpha \leq \xi<\beta\}$. An ordinal $\alpha$ with $\omega^{\alpha}=\alpha$ is called an $\varepsilon$-number. $\alpha \# \beta$ denotes the natural sum of $\alpha$ and $\beta$, in particular $\omega^{\alpha_{1}} \# \ldots \# \omega^{\alpha_{n}}=\omega^{\alpha_{p(1)}}+\ldots+\omega^{\alpha_{p(n)}}$, where $p$ is a permutation of $\{1, \ldots, n\}$ with $\alpha_{p(1)} \geq \ldots \geq \alpha_{p(n)}$.

\section{An intermediate Proof System}

In this section we introduce an intermediate infinitary proof system $\mathrm{RS}^{\star}$ which is just strong enough to prove all axioms of $\mathrm{KPi}$. In section 3 we will embed $\mathrm{RS}^{\star}$ into another infinitary system $\mathrm{RS}^{\infty}$ which so to speak is the main system of this paper and for which we will prove a cut-elimination and collapsing theorem. The main advantage of $\mathrm{RS}^{\star}$ is that here we need not to keep control over the lengths of derivations, since the complexity of the endsequent of a derivation $d$ always provides a sufficiently good upper bound for the length of $d$. Before starting with $\mathrm{RS}^{\star}$ let's give the complete list of $\mathrm{KPi}$-axioms.

\section{Axioms of $\mathrm{KPi}$}

(Ext) $\quad \forall x \forall y \forall z[x=y \rightarrow(x \in z \rightarrow y \in z) \wedge(\operatorname{Ad}(x) \rightarrow A d(y))]$

(Found) $\forall \vec{z}[\forall x(\forall y \in x \phi(y, \vec{z}) \rightarrow \phi(x, \vec{z})) \rightarrow \forall x \phi(x, \vec{z})]$

(Pair) $\quad \forall x \forall y \exists z(x \in z \wedge y \in z)$

(Union) $\forall x \exists z \forall y \in x \forall u \in y(u \in z)$ 
$\left(\Delta_{0}\right.$-Sep) $\forall \vec{z} \forall w \exists y[\forall x \in y(x \in w \wedge \phi(x, \vec{z})) \wedge \forall x \in w(\phi(x, \vec{z}) \rightarrow x \in y)]\left(\phi \in \Delta_{0}\right)$

$\left(\Delta_{0}\right.$-Col) $\forall \vec{z} \forall w\left[\forall x \in w \exists y \phi(x, y, \vec{z}) \rightarrow \exists w_{1} \forall x \in w \exists y \in w_{1} \phi(x, y, \vec{z})\right] \quad\left(\phi \in \Delta_{0}\right)$

(Ad.1) $\forall x[\operatorname{Ad}(x) \rightarrow \operatorname{tran}(x) \wedge \exists w \in x$ infinite $(w)]$

(Ad.2) $\forall x \forall y[\operatorname{Ad}(x) \wedge A d(y) \rightarrow(x \in y \vee x=y \vee y \in x)]$

(Ad.3) $\forall x\left[\operatorname{Ad}(x) \rightarrow \psi^{x}\right], \quad$ for every instance $\psi$ of

$(\operatorname{Lim}) \quad \forall x \exists y(\operatorname{Ad}(y) \wedge x \in y)$

(Pair),(Union $),\left(\Delta_{0}\right.$-Sep $),\left(\Delta_{0}\right.$ - Col $)$

The system $\mathrm{KPi}$ without $\left(\Delta_{0}-\mathrm{Col}\right)$ is called $\mathrm{KP} \ell$.

\section{Definition 2.1}

1. For each sequent $\Gamma=\left(A_{1}, \ldots, A_{n}\right)$ we define its norm $\|\Gamma\|$ by

$$
\|\Gamma\|:=\omega^{\mathrm{rk}\left(A_{1}\right)} \# \ldots \# \omega^{\mathrm{rk}\left(A_{n}\right)} .
$$

2. For $X \subseteq$ On we set $X^{\star}:=X \cup\{\omega\} \cup\{\xi+1: \xi \in X\} \cup\left\{\xi^{\mathrm{R}}: \xi \in X\right\}$.

Definition 2.2 (The infinitary system $\mathrm{RS}^{\star}$ )

We define $\mathrm{RS}^{\star}$ as the collection of all derivations (i.e. wellfounded trees of RS-sequents $\Gamma$ ) generated by the following five inference rules (where the last two are just axiom schemes):

$(\bigwedge)^{*} \frac{\ldots \Gamma, A_{\iota} \ldots(\iota \in J)}{\Gamma, \Lambda\left(A_{\iota}\right)_{\iota} \in J}$

$(\bigvee)^{\star} \frac{\Gamma \cdot A_{\iota_{0}}, \ldots, A_{\iota_{n}}}{\Gamma, \bigvee\left(A_{\iota}\right)_{\iota \in J}} \quad$ if $\iota_{0}, \ldots, \iota_{n} \in J$ and $k\left(\iota_{0}, \ldots, \iota_{n}\right) \subseteq \mathrm{k}\left(\Gamma, \bigvee\left(A_{\iota}\right)_{\iota \in J}\right)^{\star}$

$(\mathrm{Ad})^{\star} \frac{\ldots \Gamma, B\left(\mathrm{~L}_{\kappa}\right) \ldots(\kappa \leq|a|)}{\Gamma, A d(a) \rightarrow B(a)}$

$(\mathrm{Ref})^{\star} \quad \Gamma, A \rightarrow \exists z \in \mathrm{L}_{\kappa} A^{(z, \kappa)} \quad$ if $A \in \Sigma(\kappa)$ and $\kappa \in \mathrm{R}$

(Found $)^{\star} \quad \Gamma, \exists x \in \mathrm{L}_{\alpha}(\forall y \in x A(y) \wedge \neg A(x)), \forall x \in \mathrm{L}_{\alpha} A(x)$

In $\mathrm{RS}^{\star}$ we identify sequents which differ only with respect to the order of their elements. So actually we are working with multisets of RS-sentences. The formula $B(a)$ in $(A d)^{\star}$ is called the principal formula of the respective inference. 


\section{Remarks}

1. Note that every formula $A \simeq \wedge\left(A_{\iota}\right)_{\iota \in J}$ with $J=\emptyset$ (e.g. $\left.A \equiv a \notin \mathrm{L}_{0}\right)$ is derivable in $\mathrm{RS}^{\star}$ simply by an application of $(\Lambda)^{\star}$.

2. Note that in $(\mathrm{V})^{\star}$ some of the formulas $A_{t_{0}}, \ldots, A_{\iota_{n}}$ may be identical, so that for example $\frac{\Gamma, A, A, B, B, B}{\Gamma, A \vee B}$ and $\frac{\Gamma \cdot B, B}{\Gamma, A \vee B}$ are instances of $(\mathrm{V})^{\star}$.

3. If $\Gamma^{\prime}$ is a premise of an $\mathrm{RS}^{\star}$-inference with conclusion $\Gamma$ then $\left\|\Gamma^{\prime}\right\|<\|\Gamma\|$.

\section{Definition 2.3}

$\mid \star \Gamma: \Longleftrightarrow\left\{\begin{array}{l}\text { there exists an } \mathrm{RS}^{\star} \text {-derivation } d \text { of } \Gamma \text { such that } \\ \text { i) } \operatorname{rk}(B(a))<\rho \text { holds for every } \\ \text { principal formula } B(a) \text { of an (Ad) })^{\star} \text {-inference in } d \\ \left.\text { ii) if } \rho=0 \text { then } d \text { contains no application of (Ref })^{\star} \text { or (Found }\right)^{\star}\end{array}\right.$

$1 \star \Gamma: \Longleftrightarrow \mid \frac{\hbar}{0} \Gamma$

(So $\left.\right|^{\star} \Gamma$ means that $\Gamma$ is derivable by means of $(\Lambda)^{\star}$ and $(V)^{\star}$ alone.)

Lemma 2.4 (Derived rules of $\mathrm{RS}^{\star}$ )

(Weak) $\left|\star \frac{\rho}{\rho} \Gamma\right| \frac{\star}{\rho} \Gamma, C$

$(\wedge / \vee) \quad \forall \iota \in J\left(\mid \star \frac{\hbar}{\rho} \Gamma, A_{\iota}, B_{\iota}\right) \& J \subseteq J^{\prime} \Longrightarrow \mid \frac{\star}{\rho} \Gamma, \wedge\left(A_{\iota}\right)_{\iota \in J}, \bigvee\left(B_{\iota}\right)_{\iota \in J^{\prime}}$

(TND) $\quad \mid \star \neg A, A$

(TND') $\left|\star \frac{\hbar}{\rho} \Gamma, B \Longrightarrow\right| \star \frac{\hbar}{\rho} \Gamma, \neg A, A \wedge B$

$\left(\forall^{\beta}\right) \quad \forall t \in \mathcal{T}_{\beta}\left(\mid \frac{\hbar}{\rho} \Gamma, A(t)\right) \Longrightarrow|\star| \frac{\hbar}{\rho} \Gamma, \forall x \in \mathrm{L}_{\beta} A(x)$

$\left(\exists^{\beta}\right) \quad\left|\frac{\hbar}{\rho} \Gamma, A(t) \quad \& \quad t \in \mathcal{T}_{\beta} \quad \& \mathrm{k}(t) \subseteq \mathrm{k}(\Gamma, A(x))^{\star} \Longrightarrow\right| \frac{\star}{\rho} \Gamma, \exists x \in \mathrm{L}_{\beta} A(x)$

Proof. The proofs are almost trivial. We just give some short hints.

ad $(\wedge / \vee)$ : Here one uses the fact that $\mathrm{k}(\iota) \subseteq \mathrm{k}\left(A_{\iota}\right)$.

ad (TND): This is proved by transfinite induction on $\operatorname{rk}(A)$ using $(\Lambda / \bigvee)$.

ad $\left(\exists^{\beta}\right)$ : We have $\exists x \in \mathrm{L}_{\beta} A(x) \simeq \bigvee\left(t \stackrel{\circ}{\epsilon} \mathrm{L}_{\beta} \wedge A(t)\right)_{t \in \tau_{\beta}}$ with

$\left(t \stackrel{\circ}{\epsilon} \mathrm{L}_{\beta}\right) \equiv\left(t \notin \mathrm{L}_{0}\right) \simeq \Lambda(\ldots)_{\iota \in \emptyset}$.

\section{Lemma 2.5}
a) $\left.\right|^{\star} b \notin b$
b) $\mid \star a \subseteq a$
c) $\mid \star b \stackrel{\circ}{\notin} a, b \in a$, if $|b|<|a|$.
d) $\left.\right|^{\star} a \neq b, b=a$ 
e) $\mid \star a \stackrel{\circ}{\in} \mathrm{L}_{\beta}$ and $\mid \star a \in \mathrm{L}_{\beta}$, if $|a|<\beta$

f) $\star \star \operatorname{tran}\left(\mathrm{L}_{\alpha}\right)$

g) $\Vdash \exists x \in \mathrm{L}_{\alpha}$ infinite $(x)$, if $\alpha>\omega$

h) $\left.\right|^{\star} \operatorname{Ad}\left(\mathrm{L}_{\kappa}\right)$, for every $\kappa \in \mathrm{R}$.

Proof.

a) This is proved by transfinite induction on $\operatorname{rk}(b)$ as follows. By I.H. (induction hypothesis) we have $\left.\right|^{\star} t \notin t$ for all $t \in \mathcal{T}_{|b|}$. From this we obtain $\left.\right|^{\star} t \stackrel{\circ}{\notin} b, t \stackrel{\circ}{\epsilon} b \wedge t \notin t$ (by (TND')) and then $\left.\right|^{\star} t \stackrel{\circ}{\notin} b, \exists x \in b(x \notin t)$ (by $\left.(\mathrm{V})^{\star}\right)$.

Now by two more applications of $(\mathrm{V})^{\star}$ we get $\mid \star t \stackrel{\circ}{\epsilon} b \rightarrow t \neq b \quad\left(\forall t \in \mathcal{T}_{|b|}\right)$, and then $\left.\left(\right.$ by $\left.(\Lambda)^{\star}\right) \quad\right|^{\star} b \notin b$.

From now on such simple proofs will be given in a more condensed form, namely just by a (horicontal or vertical) sequence of statements ' $\mid \frac{\star}{(\rho)} \Gamma$ ' such that every but the first statement in the sequence follows from its immediate predecessor(s) by means of one or two instances of the rules $(\Lambda)^{\star},(V)^{\star},(\mathrm{Ad})^{\star}$, $(\text { Ref })^{\star},($ Weak $),(\bigwedge / \vee),(T N D),\left(T_{N D}^{\prime}\right),\left(\forall^{\beta}\right),\left(\exists^{\beta}\right)$. The above proof would then look like this:

(I.H.) $\left.\right|^{\star} t \notin t||^{\star} t \stackrel{\circ}{\notin} b, t \stackrel{\circ}{\epsilon} b \wedge t \notin t||^{\star} t \stackrel{\circ}{\notin} b, \exists x \in b(x \notin t)||^{\star} t \stackrel{\circ}{\notin} b, t \neq b$

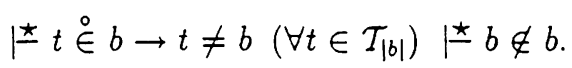

b) Induction on $\operatorname{rk}(a)$ : (I.H.) $|\star b \subseteq b| \star b=b \mid \star b \stackrel{\circ}{\notin} a, b \stackrel{\circ}{\in} a \wedge b=b$

$\mid \star b) \stackrel{\circ}{\notin} a, b \in a\left(\forall b \in \mathcal{T}_{|a|}\right) \mid \star \forall x \in a(x \in a)$.

c) This follows from the above proof of $b$ ).

d) Obvious.

e) Since $\left(a \notin \mathrm{L}_{0}\right) \simeq \wedge\left(A_{\iota}\right)_{\iota \in \emptyset}$, we have $\left.\right|^{\star} a \notin \mathrm{L}_{0}$, i.e. $\left.\right|^{\star} a \stackrel{\circ}{\epsilon} \mathrm{L}_{\beta}$. From this and $\mathrm{b})$ we obtain $\mid \star a \stackrel{\circ}{\in} \mathrm{L}_{\beta} \wedge a=a$ and then by $\left.(\mathrm{V})^{\star}\right|^{\star} a \in \mathrm{L}_{\beta}$.

f) $\left|\star t \in \mathrm{L}_{\alpha}\left(\forall b \in \mathcal{T}_{\alpha}, t \in \mathcal{T}_{|b|}\right) \quad\right| \star \forall x \in \mathrm{L}_{\alpha} \forall y \in x\left(y \in \mathrm{L}_{\alpha}\right)$.

g) $\left.\left|\star b \in \mathrm{L}_{|b|+1}\right|^{\star} \exists z \in \mathrm{L}_{\omega}(b \in z)\left(\forall b \in \mathcal{T}_{\omega}\right)\right|^{\star} \forall y \in \mathrm{L}_{\omega} \exists z \in \mathrm{L}_{\omega}(y \in z)$

$\left.\right|^{\star}$ infinite $\left.\left(L_{\omega}\right) \quad\right|^{\star} \exists w \in L_{\alpha}$ infinite $(w)$. Note that $k\left(L_{\omega}\right)=\{\omega\} \subseteq k(\ldots)^{\star}$.

h) $\left|\star L_{\kappa}=L_{\kappa} \quad\right|^{\star} A d\left(L_{\kappa}\right)$. 


\section{Abbreviation}

$\Gamma,[a \neq b]: \equiv \Gamma, \neg a \subseteq b, \neg b \subseteq a$

\section{Lemma 2.6}

$\mid \star[a \neq b],\left[\mathrm{L}_{\gamma} \neq a\right]$, if $|b|<\gamma$

Proof by induction on $\gamma$. Let $\alpha:=|a|, \beta:=|b|<\gamma$.

$\vDash \star s \neq t],\left[\mathrm{L}_{\beta} \neq s\right]$ (by I.H.)

$\left.\right|^{\star} t \stackrel{\circ}{\in} b \rightarrow t \neq s,\left[\mathrm{~L}_{\beta} \neq s\right] \quad\left(\forall t \in \mathcal{T}_{\beta}\right)$

$\vDash \star s \notin b,\left[L_{\beta} \neq s\right]$

| $s \stackrel{\circ}{\epsilon} a \wedge s \notin b, s \stackrel{\circ}{\notin} a, s \neq \mathrm{L}_{\beta}$

$\Vdash \star \exists x \in a(x \notin b), s \stackrel{\circ}{\notin} a, s \neq \mathrm{L}_{\beta} \quad\left(\forall s \in \mathcal{T}_{\alpha}\right)$

$H^{\star}[a \neq b], \mathrm{L}_{\beta} \notin a$

$\star \star[a \neq b], \exists x \in \mathrm{L}_{\gamma}(x \notin a)$

$\stackrel{\star}{\star}[a \neq b],\left[\mathrm{L}_{\gamma} \neq a\right]$

\section{Lemma 2.7}

If $A\left(x_{1}, \ldots, x_{n}\right)$ is an $R S$-formula such that every $x_{i}(i=1, \ldots n)$ has at most one free occurrence in $A(\vec{x})$ then

$$
\stackrel{\star}{H^{\prime}}\left[s_{1} \neq t_{1}\right], \ldots,\left[s_{n} \neq t_{n}\right], \neg A\left(s_{1}, \ldots, s_{n}\right), A\left(t_{1}, \ldots t_{n}\right)
$$

\section{Corollary}

$\Vdash s \neq t, \neg A(s), A(t), \quad$ for every $R S$-formula $A(x)$.

Proof of the corollary.

Given $A(x)$, there is a formula $B\left(x_{1}, \ldots, x_{n}\right)$ such that $A(x) \equiv B(x, \ldots, x)$ and every $x_{i}$ occurs at most once in $B\left(x_{1}, \ldots, x_{n}\right)$. By the lemma we have $\left.\right|^{\star}[s \neq t], \ldots,[s \neq t], \neg B(s, \ldots, s), B(t, \ldots, t)$, and from this we get, $\vDash \star s \neq t, \neg A(s), A(t)$ by $(\mathrm{V})^{\star}$.

Proof of the lemma by induction on $\operatorname{rk}(A(\vec{s})) \# \operatorname{rk}(A(\vec{t}))$

(CASE 1) $A\left(x_{1}, x_{2}\right) \equiv x_{1} \in x_{2}$.

$\left.\star \star s_{1} \neq t_{1}\right],[s \neq t], s \neq s_{1}, t=t_{1} \quad$ (by I.H.)

$\left.|\star| s_{1} \neq t_{1}\right], t \stackrel{\circ}{\notin} t_{2} \vee t \neq s, s \neq s_{1}, t \stackrel{\circ}{\epsilon} t_{2} \wedge t=t_{1} \quad\left(\forall t \in \mathcal{T}_{\left|t_{2}\right|}\right.$ 
$I^{\star}\left[s_{1} \neq t_{1}\right], s \notin t_{2}, s \neq s_{1}, t_{1} \in t_{2}$

$\mid \star\left[s_{1} \neq t_{1}\right], s \stackrel{\circ}{\epsilon} s_{2} \wedge s \notin t_{2}, s \stackrel{\circ}{\notin} s_{2} \vee s \neq s_{1}, t_{1} \in t_{2} \quad\left(\forall s \in \mathcal{T}_{\left|s_{2}\right|}\right)$

$\left.\vDash \star s_{1} \neq t_{1}\right],\left[s_{2} \neq t_{2}\right], s_{1} \notin s_{2}, t_{1} \in t_{2}$

(CASE 2) $A(x) \equiv A d(x)$.

$H^{\star}[s \neq t], L_{\kappa} \neq s, L_{\kappa}=t \quad(\forall \kappa \leq \min \{|s|,|t|\}) \quad$ (by I.H.)

$\left.\right|^{\star}[s \neq t], L_{\kappa} \neq s, A d(t) \quad(\forall \kappa \leq \min \{|s|,|t|\})$

$\left.\right|^{\star}[s \neq t], L_{\kappa} \neq s, A d(t) \quad(\forall \kappa \leq|s|) \quad\left[\right.$ by $\left.2.6 \mid \star[s \neq t], L_{\kappa} \neq s(\forall \kappa>|t|)\right]$

$\vDash \star$ $[s \neq t], \neg A d(s), A d(t)$.

(CASE 3) $A(\vec{x}) \equiv \exists y \in x_{1} B\left(x_{2}, \ldots, x_{n}, y\right)$ : similar to CASE 1 .

The remaining cases are easy.

\section{Lemma 2.8}

$\leftleftarrows b \notin a, b \stackrel{\circ}{\epsilon} a$.

Proof.

$1 \star t \stackrel{\circ}{\notin} a, t \neq b, b \stackrel{\circ}{\epsilon} a \quad($ by 2.7$)$

$\mid \star t \stackrel{\circ}{\notin} a \vee t \neq b, b \stackrel{\circ}{\in} a \quad\left(\forall t \in \mathcal{T}_{|a|}\right)$

^ $b \notin a, b \stackrel{\circ}{\epsilon} a$

\section{Theorem 2.9}

a) For every limit ordinal $\lambda$ we have $\|^{\star}(\text { Ext })^{\lambda} \wedge(\text { Found })^{\lambda} \wedge(\text { Pair })^{\lambda} \wedge(\text { Union })^{\lambda} \wedge\left(\Delta_{0}-\text { Sep }\right)^{\lambda}$

b) For every $\kappa \in R$ we have $\mid \frac{\star}{1}\left(\Delta_{0} \text {-Col }\right)^{\kappa}$.

c) For every limit ordinal $\lambda$ such that $\forall \alpha<\lambda \exists \kappa \in \mathrm{R}(\alpha<\kappa<\lambda)$ we have $\left.\right|_{\lambda} ^{\star}(\mathrm{KP} \ell)^{\lambda}$, i.e. $\left.\right|_{\lambda} ^{\star} \phi^{\lambda}$ holds for every axiom $\phi$ of $\mathrm{KP} \ell$.

Proof.

a) $($ Ext):

By 2.7 we have $\left.\right|^{\star} a \neq b, a \notin c, b \in c$ and $\left.\right|^{\star} a \neq b, \neg A d(a), A d(b), \quad$ for all $a, b, c \in \mathcal{T}$. Hence $\left.\right|^{\star}(\text { Ext })^{\lambda}$.

(Found): trivial.

(Pair):

Let $a, b \in \mathcal{T}_{\lambda}$ and $\delta:=\max \{|a|,|b|\}+1$

Then ${ }^{\star} a \in \mathrm{L}_{\delta} \wedge b \in \mathrm{L}_{\delta}$ from which we get $\left.\right|^{\star} \exists z \in \mathrm{L}_{\lambda}(a \in z \wedge b \in z)$, since 
$\delta \in(\mathrm{k}(a) \cup \mathrm{k}(b))^{\star}$ and $\delta<\lambda$.

(Union):

For every $a \in \mathcal{T}_{\lambda}$ we have

$\mid \star s \in L_{|a|} \quad\left(\forall s \in \mathcal{T}_{|a|}\right)$

$\mid \star \forall x \in t\left(x \in L_{|a|}\right) \quad\left(\forall t \in \mathcal{T}_{|a|}\right)$

$\left\lfloor\star \forall y \in a \forall x \in y\left(x \in \mathrm{L}_{|a|}\right)\right.$

$\vDash \exists \exists \in L_{\lambda} \forall y \in a \forall x \in y(x \in z)$

$\left(\Delta_{0}\right.$-Sep):

Let $\phi\left(x, z_{1}, \ldots, z_{n}\right) \in \Delta_{0}$ and $a, c_{1}, \ldots, c_{n} \in \mathcal{T}_{\lambda}$. We have to prove

$\vdash^{\star} \exists y \in \mathrm{L}_{\lambda}\left(\psi_{1}(y, a, \vec{c}) \wedge \psi_{2}(y, a, \vec{c})\right)$,

where $\dot{\psi}_{1}(y, a, \vec{c}): \equiv \forall x \in y(x \in a \wedge \phi(x, \vec{c}))$

and $\psi_{2}(y, a, \vec{c}): \equiv \forall x \in a(\phi(x, \vec{c}) \rightarrow x \in y)$.

For this let $\delta:=\max \left\{|a|,\left|c_{1}\right|, \ldots,\left|c_{n}\right|\right\}+1$, and $d:=\left[x \in \mathrm{L}_{\delta}: x \in a \wedge \phi(x, \vec{c})\right]$.

Then $d \in \mathcal{T}_{\lambda}$ and $\mathrm{k}(d) \subseteq \mathrm{k}\left(\psi_{1}(y, a, \vec{c})\right)^{\star}$.

Therefore it suffices to prove (1) $\left.\quad\right|^{\star} \psi_{1}(d, a, \vec{c})$ and (2) $\left.\quad\right|^{\star} \psi_{2}(d, a, \vec{c})$.

But (1) follows immediately from the fact that $t \stackrel{\circ}{\epsilon} d \equiv t \in a \wedge \phi(t, \vec{c})$ and therefore $\mid \star t \stackrel{\circ}{\in} d \rightarrow t \in a \wedge \phi(t, \vec{c}) \quad\left(\forall t \in \mathcal{T}_{\delta}\right)$.

And (2) is obtained as follows:

$\leftleftarrows t \stackrel{\circ}{\notin} a, t \in a$

$\mu^{\star} t \dot{\notin} a, \neg \phi(t, \vec{c}), t \in a \wedge \phi(t, \vec{c})$

$\mu^{\star} t \stackrel{\circ}{\notin} a, \neg \phi(t, \vec{c}), t \stackrel{\circ}{\in} d \wedge t=t$

$\mid \star t \stackrel{\ddagger}{\notin} a, \neg \phi(t, \vec{c}), t \in d \quad\left(\forall t \in \mathcal{T}_{|a|}\right)$

$\mu^{\star} \forall x \in a(\phi(x, \vec{c}) \rightarrow x \in d)$

b) is an immediate consequence of (Ref) ${ }^{\star}$.

c) 1 . If $\phi$ is an instance of (Ext),(Found),(Pair),(Union), $\left(\Delta_{0}\right.$-Sep), then $\left.\right|^{\star} \phi^{\lambda}$ holds by a).

2. Suppose that $\phi$ is an axiom (Ad1) or (Ad3). Then $\phi \equiv \forall x(A d(x) \rightarrow \chi(x))$ with $\chi(x) \in \Delta_{0}$, and by $2.5 \mathrm{f}, \mathrm{g}$ and $2.9 \mathrm{a}$, b we have $\mid \frac{\star}{1} \chi\left(\mathrm{L}_{\kappa}\right)$ for all $\kappa \in \mathrm{R}$. Since $\forall a \in \mathcal{T}_{\lambda}(\operatorname{rk}(\chi(a))<\omega \lambda=\lambda)$, by $(\operatorname{Ad})^{\star}$ we get $\forall a \in \mathcal{T}_{\lambda}\left(\left.\right|_{\lambda} ^{\star} A d(a) \rightarrow \gamma(a)\right)$ and thus $\mid \frac{\hbar}{\lambda} \phi^{\lambda}$. 
3. In the same way as under 2. we obtain $\left.\right|_{\lambda} ^{\star}(\operatorname{Ad} 2)^{\lambda}$.

4. Now we prove $\left.\right|^{\star}(\operatorname{Lim})^{\lambda}$. Let $a \in \mathcal{T}_{\lambda}$ and $\kappa:=|a|^{R}$. Then $|a|<\kappa<\lambda$, $\kappa \in \mathrm{k}\left(\exists y \in \mathrm{L}_{\lambda}(\operatorname{Ad}(y) \wedge a \in y)\right)^{\star}$ and $\left.\right|^{\star} \operatorname{Ad}\left(\mathrm{L}_{\kappa}\right) \wedge a \in \mathrm{L}_{\kappa}$ (by 2.5). From this we get $\left.\right|^{\star} \exists y \in \mathrm{L}_{\lambda}(A d(y) \wedge a \in y)$ by $\left(\exists^{\lambda}\right)$.

\section{$3 \quad \mathcal{H}$-controlled derivations}

In this section we introduce the infinitary proof system $\mathrm{RS}^{\infty}$ and the notion of an $\mathcal{H}$-controlled $\mathrm{RS}^{\infty}$-derivation. We then prove that every $\mathrm{RS}^{\star}$-derivation can be transformed into an $\mathcal{H}$-controlled $\mathrm{RS}^{\infty}$-derivation and that the class of $\mathcal{H}$-controlled $\mathrm{RS}^{\infty}$-derivations is closed under predicative cut-elimination.

Definition 3.1 (The infinitary system $\mathrm{RS}^{\infty}$ )

We define $\mathrm{RS}^{\infty}$ as the collection of all derivations (i.e. wellfounded trees of pairs $\Gamma: \alpha)$ generated by the following inference rules
$(\Lambda) \quad \frac{\ldots \Gamma, A_{\iota}: \alpha_{\iota} \ldots(\iota \in J)}{\Gamma, \Lambda\left(A_{\iota}\right)_{\iota \in J}: \alpha} \quad\left(\alpha_{\iota}<\alpha\right)$
(V) $\frac{\Gamma, A_{\iota_{0}}: \alpha_{0}}{\Gamma, \bigvee\left(A_{\iota}\right)_{\iota \in J}: \alpha} \quad\left(\alpha_{0}<\alpha, \iota_{0} \in J,\left|\iota_{0}\right|<\alpha\right)$
(Cut) $\frac{\Gamma, \neg C: \alpha_{0} \quad \Gamma, C: \alpha_{0}}{\Gamma: \alpha} \quad\left(\alpha_{0}<\alpha\right)$
(Ref) $\frac{\Gamma, A: \alpha_{0}}{\Gamma, \exists z \in \mathrm{L}_{\kappa} A^{(z, \kappa)}: \alpha} \quad\left(\alpha_{0}+1<\alpha, A \in \Sigma(\kappa)\right)$

In $\mathrm{RS}^{\infty}$ we identify every RS-sequent $\Gamma=\left(A_{1}, \ldots, A_{n}\right)$ with its underlying set $\left\{A_{1}, \ldots, A_{n}\right\}$, so that for example $\frac{\Gamma \cdot A \vee B, A: \alpha}{\Gamma, A \vee B: \alpha+1}$ is an instance of $(\mathrm{V})$.

The cut-rank of an $\mathrm{RS}^{\infty}$-derivation $d$ is defined as the least ordinal $\rho$ such that $\operatorname{rk}(C)<\rho$ for all cut-formulas $C$ in $d$.

If $\Gamma: \alpha$ is the bottommost pair of $d \in \mathrm{RS}^{\infty}$ we call $d$ a derivation of $\Gamma: \alpha$ or a derivation of $\Gamma$ with ordinal $\alpha$.

We write $\mid \frac{\alpha}{\rho} \Gamma$ to express that there exists an $\mathrm{RS}^{\infty}$-derivation of $\Gamma: \alpha$ with cut-rank $\leq \rho$.

According to Lemma 1.7 the rules $(\Lambda),(V),($ Cut $)$ are correct with respect to our standard semantics of $\mathcal{L}_{R S}$. This gives us the following lemma. 


\section{Lemma 3.2 (Truth-Lemma)}

$\kappa=\min (R) \& k(\Gamma) \subseteq \kappa \& \mid \frac{\alpha}{\kappa} \Gamma \Longrightarrow \vDash \Gamma$

Note that - apart from the restrictions ' $\left|\iota_{0}\right|<\alpha$ ' in $(V)$ and ' $\alpha_{0 \pm 1}<\alpha$ ' in (Ref) - the just defined notion of $\mathrm{RS}^{\infty}$-derivation is completely standard. Therefore, according to (Tait [20]), $\mathrm{RS}^{\infty}$ allows predicative cut-elimination, i.e. every derivation of $\Gamma: \beta$ with cut-rank $\leq \omega^{\alpha}$ can be transformed into a derivation of $\Gamma: \varphi \alpha \beta$ where all cut-formulas are of the form ${ }_{\forall}^{\exists} z \in \mathrm{L}_{\kappa} A^{(z, \kappa)}$

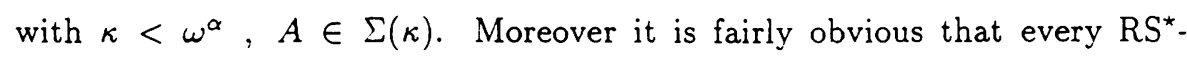
derivation of $\Gamma$ can be transformed into an $\mathrm{RS}^{\infty}$-derivation of $\Gamma:\|\Gamma\|$. But of course these facts are not sufficient to establish nontrivial upper bounds for the proof theoretic ordinals of $\mathrm{KPi}, \mathrm{KP} \ell$ or similar theories. In order to get such bounds we introduce the concept of $\mathcal{H}$-controlled $\mathrm{RS}^{\infty}$-derivations. Compared to the already existing methods this concept has the great advantage of being entirely independent from any system of collapsing functions or ordinal notations. Collapsing functions are now localized very sharply just to that part of the story where they really show up in the formulation of the result(s), i.e. the Collapsing Theorem.

We continue with some preliminaries to the definition of $\mathcal{H}$-controlled $\mathrm{RS}^{\infty}$ derivations. Let $\mathrm{SEQ}$ be the class of all RS-sequents. We identify each $\mathrm{RS}^{\infty}$. derivation in the usual way with a function $d: \operatorname{dom}(d) \longrightarrow \mathrm{SEQ} \times$ On where $\operatorname{dom}(d)$ is a subset of $\left\{\left\langle\iota_{0}, \ldots, \iota_{n-1}\right\rangle: n \in \omega \& \iota_{0}, \ldots, \iota_{n-1} \in \mathcal{T}^{0,1}\right\}$ closed under initial segments. The elements of $\operatorname{dom}(d)$ are called the nodes of $d$, and the empty sequence \langle\rangle$\in \operatorname{dom}(d)$ is called the bottom node or root of $d$. For $s \in \operatorname{dom}(d)$ and $d(s)=(\Gamma: \alpha)$ we call $\Gamma(\alpha$, resp.) the sequent (ordinal, resp.) at node $s$, and set $\mathrm{k}(d(\mathrm{~s})):=\mathrm{k}(\Gamma) \cup\{\alpha\}$.

(To avoid a possible misunderstanding we point out that the index of the premise $\Gamma, A_{\iota_{0}}$ of an $(\mathrm{V})$-inference is 0 and not at all $\iota_{0}$. So, if the conclusion of an $(\mathrm{V})$-inference is at node $s$, then its premise is at node $s *\langle 0\rangle$.)

\section{Definition 3.3 ( $\mathcal{H}$-controlled $\mathrm{RS}^{\infty}$-derivations)}

Functions $\mathcal{H}: \mathcal{P}(\mathrm{On}) \longrightarrow \mathcal{P}(\mathrm{On})$ are henceforth called operators.

Let $\mathcal{H}$ be an operator, and $d: \operatorname{dom}(d) \longrightarrow \mathrm{SEQ} \times \mathrm{On}$ an $\mathrm{RS}^{\infty}$-derivation.

We say that $d$ is $\mathcal{H}$-controlled if, and only if

$$
k(d(s)) \subseteq \mathcal{H}(k(s)) \quad \text { for all } s \in \operatorname{dom}(d)
$$


The intuitive idea behind this definition is that, for each node $\mathrm{s}$ of $d, \mathcal{H}$ tells us which ordinals are allowed (or available) at $s$.

\section{Definition 3.4}

Let $\mathcal{H}$ be an operator and $\Theta$ a finite sequence of RS-sentences and elements of $\mathcal{T}^{0,1}$. Then we define the operator $\mathcal{H}[\Theta]: \mathcal{P}(\mathrm{On}) \longrightarrow \mathcal{P}(\mathrm{On})$ by

$$
\mathcal{H}[\Theta](X):=\mathcal{H}(\mathrm{k}(\Theta) \cup X)
$$

\section{Abbreviations}

Let $\mathcal{H}$ be an operator and $f$ some ordinal function.

$\alpha \in \mathcal{H}: \Leftrightarrow \alpha \in \mathcal{H}(\emptyset)$

$X \subseteq \mathcal{H}: \Leftrightarrow X \subseteq \mathcal{H}(\emptyset)$

$\mathcal{H} \subseteq X: \Leftrightarrow \mathcal{H}(\emptyset) \subseteq X$

$\mathcal{H}$ is closed under $f: \Leftrightarrow \forall X \in \mathcal{P}(\mathrm{On})[\mathcal{H}(X)$ is closed under $f]$

\section{Remarks}

1. Note that ' $\alpha \in \mathcal{H}[\Theta]$ ' (' $X \subseteq \mathcal{H}[\Theta]$ ', resp.) is synonymous with ' $\alpha \in$ $\mathcal{H}(\mathrm{k}(\Theta))$ ' ('X $X \mathcal{H}(\mathrm{k}(\Theta))$ ', resp.).

2. We always have $\mathcal{H}\left[\Theta, \Theta^{\prime}\right]=\mathcal{H}[\Theta]\left[\Theta^{\prime}\right]$.

In order to come up with a smooth theory of $\mathcal{H}$-controlled derivations we will from now on restrict our considerations to operators $\mathcal{H}$ which satisfy certain minimal closure conditions. These operators will be called nice.

Definition 3.5 (Nice operators)

i) A set $X \subseteq$ On is called nice iff $0 \in X \& \forall n \in \omega \forall \alpha_{0}, \ldots, \alpha_{n}\left(\omega^{\alpha_{0}} \# \ldots \# \omega^{\alpha_{n}} \in X \Leftrightarrow\left\{\alpha_{0}, \ldots, \alpha_{n}\right\} \subseteq X\right)$

ii) An operator $\mathcal{H}$ is called nice iff the following holds for all $X, X^{\prime} \in \mathcal{P}(\mathrm{On})$ :

(H.1) $\mathcal{H}(X)$ is nice.

(H.2) $X \subseteq \mathcal{H}(X)$

(H.3) $\quad X^{\prime} \subseteq \mathcal{H}\left(X^{\prime}\right) \Longrightarrow \mathcal{H}\left(X^{\prime}\right) \subseteq \mathcal{H}(X)$

Lemma 3.6

If $\mathcal{H}$ is a nice operator then the following holds for all $\Theta$.

a) $\mathcal{H}[\Theta]$ is nice.

b) $\mathrm{k}(\Theta) \subseteq \mathcal{H} \Longrightarrow \mathcal{H}[\Theta]=\mathcal{H}$

c) $\forall X . X^{\prime} \in \mathcal{P}($ On $)\left[X^{\prime} \subseteq X \Longrightarrow \mathcal{H}\left(X^{\prime}\right) \subseteq \mathcal{H}(X)\right]$

d) $\mathcal{H}$ is closed under addition. multiplication and exponentiation to base w.

e) If $\mathcal{H}$ is closed under $\xi \mapsto \xi^{\mathrm{R}}$ then $X^{\star} \subseteq \mathcal{H}(X)$ for all $X \in \mathcal{P}(\mathrm{On})$. 


\section{Definition 3.7}

$\mathcal{H} \mid \frac{\alpha}{\rho} \Gamma: \Longleftrightarrow \exists \mathcal{H}$-controlled $\mathrm{RS}^{\infty}$-derivation of $(\Gamma: \alpha)$ with cut-rank $\leq \rho$

$$
\text { Abbreviation: } \quad \mathcal{H}\left|\frac{\alpha}{\rho} \Gamma,(\neg) C: \Longleftrightarrow \mathcal{H}\right| \frac{\alpha}{\rho} \Gamma, \neg C \& \mathcal{H} \mid \frac{\alpha}{\rho} \Gamma, C \text {. }
$$

The following Theorem provides a characterization of $\mathcal{H} \mid \frac{\alpha}{\rho} \Gamma$ by transfinite recursion on $\alpha$ which as well could be taken as the definition of $\mathcal{H} \mid \frac{\alpha}{\rho} \Gamma$. Actually in what follows we are always working with this derivability relation and not with specific derivations.

\section{Theorem 3.8}

$\mathcal{H} \mid \frac{\alpha}{\rho} \Gamma$ if, and only if, $\{\alpha\} \cup \mathrm{k}(\Gamma) \subseteq \mathcal{H}$ and one of the following cases holds:
$(\Lambda) \quad \Lambda\left(A_{\iota}\right)_{\iota \in J} \in \Gamma \quad \& \quad \mathcal{H}[\iota] \mid \frac{\alpha_{\iota}}{\rho} \Gamma, A_{\iota} \quad \& \quad \alpha_{\iota}<\alpha \quad(\forall \iota \in J)$
(V) $\quad \mathrm{V}\left(A_{\iota}\right)_{\iota \in J} \in \Gamma \quad \& \quad \mathcal{H}\left|\frac{\alpha_{0}}{\rho} \Gamma, A_{\iota 0} \quad \& \quad \alpha_{0}<\alpha \quad \& \quad \iota_{0} \in J\right| \alpha$
(Cut) $\operatorname{rk}(C)<\rho \quad \& \mathcal{H} \mid \frac{\alpha_{0}}{\rho} \Gamma,(\neg) C \quad \& \quad \alpha_{0}<\alpha$
(Ref) $\exists z \in \mathrm{L}_{\kappa} A^{(z, \kappa)} \in \Gamma \quad \& \quad \mathcal{H} \mid \frac{\alpha_{0}}{\rho} \Gamma, A \quad \& \quad \alpha_{0}+1<\alpha \quad \& \quad A \in \Sigma(\kappa)$

\section{General Assumption}

In the following $\mathcal{H}$ always denotes some nice operator.

Now we are going to prove the three main results of this section, i.e. the Embedding Theorem, the Predicative Cut-Elimination Theorem, and the Boundedness Lemma for $\mathcal{H}$-controlled $\mathrm{RS}^{\infty}$-derivations.

\section{Lemma 3.9}

a) $\mathcal{H}\left|\frac{\alpha}{\rho} \Gamma \& \alpha \leq \alpha^{\prime} \in \mathcal{H} \& \rho \leq \rho^{\prime} \& \mathrm{k}\left(\Gamma^{\prime}\right) \subseteq \mathcal{H} \Longrightarrow \mathcal{H}\right| \frac{\alpha^{\prime}}{\rho^{\prime}} \Gamma, \Gamma^{\prime}$

b) $\mathcal{H}\left|\frac{\alpha}{\rho} \Gamma, A \vee B \Longrightarrow \mathcal{H}\right| \frac{\alpha}{\rho} \Gamma, A, B$

c) $\mathcal{H}\left|\frac{\alpha}{\rho} \Gamma, \forall x \in \mathrm{L}_{\kappa} A(x) \& \beta<\kappa \& \beta \in \mathcal{H} \Longrightarrow \mathcal{H}\right| \frac{\alpha}{\rho} \Gamma, \forall x \in \mathrm{L}_{\beta} A(x)$

Proof by induction on $\alpha$.

\section{Lemma 3.10}

If $\mathcal{H}$ is closed under $\xi \mapsto \xi^{\mathrm{R}}$, then $\mid \frac{\star}{\rho} \Gamma$ implies $\mathcal{H}[\Gamma] \mid \frac{\|\Gamma\|}{\rho} \Gamma$.

Proof.

Abbreviation: $\mathcal{H}\left|\frac{\star}{\rho} \Gamma: \Leftrightarrow \mathcal{H}[\Gamma]\right| \frac{\|\Gamma\|}{\rho} \Gamma$.

We prove that $\mathcal{H} \mid \frac{\star}{\rho}$ is closed under the inference rules of $\mathrm{RS}^{\star}$. 
1. Suppose that $\Gamma=\Gamma^{\prime}, A$ with $A \simeq \wedge\left(A_{\iota}\right)_{\iota \in J}$ and $\mathcal{H} \mid \frac{\star}{\rho} \Gamma^{\prime}, A_{\iota}$ for all $\iota \in J$. Let $\alpha:=\|\Gamma\|, \alpha_{\iota}:=\left\|\Gamma^{\prime}, A_{\iota}\right\|$. Then $\alpha_{\iota}<\alpha \in \mathcal{H}[\Gamma]$ and $\mathcal{H}\left[\Gamma^{\prime}, A_{\iota}\right] \mid \frac{\alpha_{\iota}}{\rho} \Gamma^{\prime}, A_{\iota}$ for all $\iota \in J$. Since $\mathrm{k}\left(\Gamma^{\prime}, A_{\iota}\right) \subseteq \mathrm{k}(\Gamma, \iota)$, the latter implies $\mathcal{H}[\Gamma][\iota] \mid \frac{\alpha_{\iota}}{\rho} \Gamma^{\prime}, A_{\iota}$ for all $\iota \in J$. Hence $\mathcal{H}[\Gamma] \mid \frac{\alpha}{\rho} \Gamma$ by $(\Lambda)$.

2. Suppose that $\Gamma=\Gamma^{\prime}, A$ with $A \simeq \mathrm{V}\left(A_{\iota}\right)_{\iota \in J}$ and $\mathcal{H} \mid \frac{\star}{\rho} \Gamma^{\prime} . A_{\iota_{0}}, \ldots, A_{\iota_{n}}$ where $\iota_{0}, \ldots, \iota_{n} \in J$ and $\mathrm{k}\left(\iota_{0}, \ldots, \iota_{n}\right) \subseteq \mathrm{k}(\Gamma)^{\star}$. Let $\alpha:=\|\Gamma\|$ and $\alpha_{0}:=$ $\left\|\Gamma^{\prime}, A_{\iota_{0}}, \ldots, A_{\iota_{n}}\right\|$. Since $\mathrm{k}\left(\Gamma^{\prime}, A_{\iota_{0}}, \ldots, A_{\iota_{n}}\right) \subseteq \mathrm{k}\left(\Gamma, \iota_{0}, \ldots, \iota_{n}\right) \subseteq \mathrm{k}(\Gamma)^{\star} \subseteq$ $\mathcal{H}[\Gamma]$, the assumption yields $\mathcal{H}[\Gamma] \mid \frac{\alpha_{0}}{\rho} \Gamma^{\prime}, A_{\iota_{0}}, \ldots, A_{\iota_{\mathrm{n}}}$. From this and the fact that $\alpha_{0}+\omega \leq \alpha \in \mathcal{H}[\Gamma]$ we obtain $\mathcal{H}[\Gamma] \mid \frac{\alpha}{\rho} \Gamma$ by $\mathrm{n}+1$ applications of $(\mathrm{V})$. (Note that $\left|\iota_{i}\right|<\|A\| \leq\|\Gamma\|=\alpha$.)

3. Suppose that $\Gamma=\Gamma^{\prime}, A d(a) \rightarrow B(a)$ with $\operatorname{rk}(B(a))<\rho$ and $\mathcal{H} \mid \frac{\star}{\rho} \Gamma^{\prime}, B\left(\mathrm{~L}_{\kappa}\right)$ for all $\kappa \leq|a|$. Let $\alpha:=\|\Gamma\|, \alpha_{0}:=\left\|\Gamma^{\prime}, \neg A d(a), B(a), B(a)\right\|$. Then we have $\alpha_{0}, \alpha \in \mathcal{H}[\Gamma], \forall \kappa \leq|a|\left(\left\|\Gamma^{\prime}, B\left(\mathrm{~L}_{\kappa}\right)\right\|<\alpha_{0}\right)$ and $\mathcal{H}\left[\Gamma, \mathrm{L}_{\kappa}\right] \mid \frac{\alpha_{0}}{\rho} \Gamma^{\prime}, B\left(\mathrm{~L}_{\kappa}\right)$ for all $\kappa \leq|a|$. By 2.7 we have $\left.\right|^{\star} \mathrm{L}_{\kappa} \neq a, \neg B\left(\mathrm{~L}_{\kappa}\right), B(a)$. By 1 . and 2. above $\mathcal{H} \mid \frac{\star}{\rho}$ is closed under $(\Lambda)^{\star}$ and $(V)^{\star}$. Hence $\mathcal{H}\left[\Gamma, L_{\kappa}\right] \mid \frac{\alpha_{0}}{\rho} L_{\kappa} \neq a, \neg B\left(L_{\kappa}\right), B(a)$, for all $\kappa \leq|a|$, and by (Cut) we get $\mathcal{H}\left[\Gamma, L_{\kappa}\right] \mid \frac{\alpha_{0+1}}{\rho} \Gamma^{\prime}, L_{\kappa} \neq a . B(a)$, for all $\kappa \leq|a|$. This yields $\mathcal{H}[\Gamma] \mid \frac{\alpha_{0}+2}{\rho} \Gamma^{\prime}, \neg A d(a), B(a)$ and then $\mathcal{H}[\Gamma] \mid \frac{\alpha}{\rho} \Gamma^{\prime}, A d(a) \rightarrow B(a)$, since $\alpha_{0}<\alpha \in \mathrm{Lim}$.

4. Let $A \in \Sigma(\kappa)$. By 2.4 we have $\mid \star \neg A, A$ and therefore $\mathcal{H}[A] \mid \frac{\alpha_{0}}{\rho} \neg A . A$ with $\alpha_{0}:=\|\neg A, A\|$. From this by (Ref) and (V) we get $\mathcal{H}[C] \mid \frac{\|C\|}{\rho} C$ with $C: \equiv A \rightarrow \exists z \in \mathrm{L}_{\kappa} A^{(z, \kappa)}$.

5. Suppose that $\Gamma=\Gamma^{\prime}, C, \forall x \in \mathrm{L}_{\alpha} A(x)$ with $C \equiv \exists x \in \mathrm{L}_{\alpha}(\forall y \in x A(y) \wedge \neg A(x))$. Let $\gamma_{t}:=\|C\|+\omega|t|$. By induction on $|a|$ we prove $\mathcal{H}[C, a] \mid \frac{\gamma_{a}}{\rho} C, \forall x \in a A(x)$ for all $a \in \mathcal{T}$ with $|a| \leq \alpha$. This yields $\mathcal{H}[\Gamma] \mid \frac{\|\Gamma\|}{\rho} \Gamma$. since $k\left(C, \mathrm{~L}_{\alpha}\right) \subseteq k(\Gamma)$ and $\|C\|+\omega\left|\mathrm{L}_{\alpha}\right| \leq\|\Gamma\|$. So let $|a| \leq \alpha$. By I.H. we have (1) $\mathcal{H}[C, t] \mid \frac{\gamma_{t}}{\rho} C$. $\forall y \in t A(y)$ for all $t \in \mathcal{T}_{|a|}$. By 1 . and 2. above we have (2) $\mathcal{H}[A(t)] \mid \frac{\alpha_{t}}{\rho} \neg . A(t) . A(t)$ with $\alpha_{t}:=\|\neg A(t), A(t)\|$. Since $k(A(t)) \subseteq \mathrm{k}(C, t)$ and $\alpha_{t} \leq \gamma_{t}$ for $t \in \mathcal{T}_{\alpha}$, 
from (1) and (2) we obtain $\mathcal{H}[C, t] \mid \frac{\gamma_{t+2}}{\rho} C, t \stackrel{\circ}{\epsilon} \mathrm{L}_{\alpha} \wedge \forall y \in t A(y) \wedge \neg A(t), A(t)$ for all $t \in \mathcal{T}_{|a|}$. By $(\mathrm{V})$ we get $\mathcal{H}[C, t] \mid \frac{\gamma_{t}+3}{\rho} C, A(t)$ for all $t \in \mathcal{T}_{|a|}$. Hence $\mathcal{H}[C, a] \mid \frac{\gamma_{a}}{\rho} C, \forall x \in a A(x)$.

\section{Lemma 3.11}

Let $\lambda \in \mathcal{H}$. Then for every logically valid sequent $\Delta(\vec{x})$ of $\mathcal{L}_{A d}$-formulas there is an $m<\omega$ such that $\mathcal{H}[\vec{a}] \mid \frac{\omega^{\omega \lambda+m}}{\omega \lambda} \Delta^{\lambda}(\vec{a})$ for all $\vec{a} \in \mathcal{T}_{\lambda}$.

Proof. Abbreviation: $\mathcal{H} \vdash \Delta(\vec{x}): \Leftrightarrow \exists m<\omega \forall \vec{a} \in \mathcal{T}_{\lambda}\left[\mathcal{H}[\vec{a}] \mid \frac{\omega^{\omega \lambda+m}}{\omega \lambda} \Delta^{\lambda}(\vec{a})\right]$.

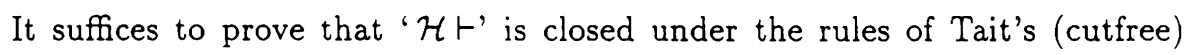
calculus for first order predicate logic.

1. By 2.4 and 3.10 for every atomic formula $\phi(\vec{x})$ and $\vec{a} \in \mathcal{T}_{\lambda}$ we have $\mathcal{H}[\vec{a}] \mid \frac{\omega^{\omega \lambda}}{0} \neg \phi^{\lambda}(\vec{a}), \phi^{\lambda}(\vec{a})$.

2. Suppose that $\forall y \phi(\vec{x}, y) \in \Delta(\vec{x})$ and $\mathcal{H} \vdash \Delta(\vec{x}), \phi(\vec{x}, y)$ with $y \notin\{\vec{x}\}$. Then for some $\alpha=\omega \lambda+m$ we have $\quad(*) \mathcal{H}[\vec{a}, b] \mid \frac{\omega^{\alpha}}{\omega \lambda} \Delta^{\lambda}(\vec{a}), \phi^{\lambda}(\vec{a}, b) \quad\left(\forall \vec{a}, b \in \mathcal{T}_{\lambda}\right)$. Let $\vec{a} \in \mathcal{T}_{\lambda}$ be fixed.

2.1. Suppose that $\forall y$ is unrestricted. Then $(\forall y \phi(\vec{a}, y))^{\lambda} \equiv \forall y \in \mathrm{L}_{\lambda} \phi^{\lambda}(\vec{a}, y)$ and from (*) we get $\mathcal{H}[\vec{a}] \mid \frac{\omega^{\alpha+1}}{\omega \lambda} \Delta^{\lambda}(\vec{a}), \forall y \in \mathrm{L}_{\lambda} \phi^{\lambda}(\vec{a}, y)$.

2.2. Suppose that $\phi(\vec{x}, y) \equiv y \in x_{i} \rightarrow \psi(\vec{x}, y)$. In this case $(\forall y \phi(\vec{a}, y))^{\lambda} \equiv$ $\forall y \in a_{i} \phi^{\lambda}(\vec{a}, y)$, and from (*) we get $\mathcal{H}[\vec{a}, b] \mid \frac{\omega^{\alpha}}{\omega \lambda} \Delta^{\lambda}(\vec{a}), b \notin a_{i}, \psi^{\lambda}(\vec{a}, b)$ for all $b \in \mathcal{T}_{\lambda}$. By $2.5 \mathrm{c}$ and 3.10 we also have $\mathcal{H}[\vec{a}, b] \mid \frac{\omega^{\alpha}}{\omega \lambda} b \in a_{i}, b \stackrel{\circ}{\notin} a_{i}$ for all $b \in \mathcal{T}_{\left|a_{i}\right|}$. By (Cut) we obtain $\mathcal{H}[\vec{a}, b] \mid \frac{\omega^{\alpha}+1}{\omega \lambda} \Delta^{\lambda}(\vec{a}), b \stackrel{\circ}{\notin} a_{i}, \psi^{\lambda}(\vec{a}, b) \quad\left(\forall b \in \mathcal{T}_{\left|a_{i}\right|}\right)$. Now we apply $(\bigvee)$ and $(\Lambda)$ and get $\mathcal{H}[\vec{a}] \mid \frac{\omega^{\alpha+1}}{\omega \lambda} \Delta^{\lambda}(\vec{a}), \forall y \in a_{i} \psi^{\lambda}(\vec{a}, y)$.

3. The case of an $\exists$-inference is treated similarly to case 2 .

4. The $\wedge$ - and $\vee$-cases are easy.

From 2.9, 3.10 and 3.11 we get the following theorem.

Theorem 3.12 (Embedding)

Suppose that $\lambda \in \mathcal{H}$ with $\lambda \in \mathrm{R} \quad \& \quad \forall \alpha<\lambda \exists \kappa \in \mathrm{R}(\alpha<\kappa<\lambda)$, and that $\mathcal{H}$ is closed under $\xi \mapsto \xi^{\mathrm{R}}$. Then for each theorem $\phi$ of $\mathrm{K}$. $i$ there is an $m<\omega$ such that $\mathcal{H} \mid \frac{\omega^{\lambda+m}}{\lambda+m} \phi^{\lambda}$. 
We now turn to the Predicative Cut-Elimination Theorem.

Lemma 3.13 (Inversion)

$\mathcal{H}\left|\frac{\alpha}{\rho} \Gamma, \wedge\left(A_{\iota}\right)_{\iota \in J} \quad \& \quad \iota_{0} \in J \Longrightarrow \mathcal{H}\left[\iota_{0}\right]\right| \frac{\alpha}{\rho} \Gamma, A_{\iota 0}$

Proof by induction on $\alpha$.

Lemma 3.14 (Reduction)

Suppose that $C \simeq \vee\left(C_{\iota}\right)_{\iota \in J}$ and $\operatorname{rk}(C)=\rho \notin \mathrm{R}$. Then the following holds:

$\mathcal{H}\left|\frac{\alpha}{\rho} \Gamma^{\prime}, \neg C \& \mathcal{H}\right| \frac{\beta}{\rho} \Gamma, C \Longrightarrow \mathcal{H} \mid \frac{\alpha+\beta}{\rho} \Gamma^{\prime}, \Gamma$.

Proof by induction on $\beta$.

We treat only the crucial case where $\Gamma, C$ is the conclusion of an $(\mathrm{V})$-inference with principal part $C$. So assume that $\mathcal{H} \mid \frac{\beta_{0}}{\rho} \Gamma, C, C_{\iota_{0}}$ with $\iota_{0} \in J \mid \beta$ and $\beta_{0}<\beta \in \mathcal{H}$. Then by I.H. we have

(1) $\mathcal{H} \mid \frac{\alpha+\beta_{0}}{\rho} \Gamma^{\prime}, \Gamma, C_{\iota_{0}}$.

We also have $\beta_{0} \in \mathcal{H}, \neg C \simeq \wedge\left(\neg C_{\iota}\right)_{\iota \in J}, \mathrm{k}\left(\iota_{0}\right) \subseteq \mathrm{k}\left(C_{\iota_{0}}\right) \subseteq \mathcal{H}$. The latter yields $\mathcal{H}\left[\iota_{0}\right]=\mathcal{H}$. Therefore by 3.13 and $3.9 \mathrm{a}$ from $\mathcal{H} \mid \frac{\alpha}{\rho} \Gamma^{\prime}, \neg C$ we get (2) $\mathcal{H} \mid \frac{\alpha+\beta_{0}}{\rho} \Gamma^{\prime}, \Gamma, \neg C_{\iota_{0}}$. Now we apply (Cut) to (1),(2) and obtain $\mathcal{H} \mid \frac{\alpha+\beta}{\rho} \Gamma^{\prime}, \Gamma, \quad$ since $\operatorname{rk}\left(C_{\iota_{0}}\right)<\operatorname{rk}(C)=\rho$ and $\alpha+\beta_{0}<\alpha+\beta \in \mathcal{H}$.

Note that, since $\operatorname{rk}(C) \notin \mathrm{R}, C$ cannot be the main part of a (Ref)-inference.

Definition 3.15 (The Veblen function $\varphi$ )

$\varphi \alpha \beta:=\varphi_{\alpha}(\beta)$, where $\varphi_{\alpha}$ is defined by transfinite recursion on $\alpha$ as the ordering function of the class $\left\{\omega^{\beta}: \beta \in\right.$ On $\left.\& \forall \xi \in \alpha\left(\varphi_{\xi}\left(\omega^{\beta}\right)=\omega^{\beta}\right)\right\}$

Corollary (Basic properties of $\varphi$ )

$(\varphi .1) \varphi 0 \beta=\omega^{\beta}, \varphi 1 \beta=\varepsilon_{\beta}$

$(\varphi .2) \xi, \eta<\varphi \alpha \beta \Longrightarrow \xi+\eta<\varphi \alpha \beta$

( $\varphi .3) \quad \beta_{0}<\beta \Longrightarrow \varphi \alpha \beta_{0}<\varphi \alpha \beta$

(

Theorem 3.16 (Predicative Cut-Elimination)

If $\mathcal{H}$ is closed under the Veblen-function $\varphi$ then the following holds:

$\mathcal{H} \mid \frac{3}{\rho+\omega^{\alpha}} \Gamma \&\left[\rho, \rho+\omega^{\alpha}\left[\cap \mathrm{R}=\emptyset \& \alpha \in \mathcal{H} \Longrightarrow \mathcal{H} \mid \frac{\varphi \alpha \beta}{\rho} \Gamma\right.\right.$. 
Proof by main induction on $\alpha$ and subsidiary induction on $\beta$.

Again we only treat the crucial case.

Assume $\mathcal{H} \mid \frac{\beta_{0}}{\rho+\omega^{\alpha}}(\neg) C, \Gamma \& \beta_{0}<\beta \in \mathcal{H} \& \operatorname{rk}(C)<\rho+\omega^{\alpha}$.

Then by S.I.H. we have

(1) $\mathcal{H} \mid \frac{\varphi \alpha \beta_{0}}{\rho}(\neg) C, \Gamma$.

From $\alpha, \beta \in \mathcal{H}$ we get

(2) $\varphi \alpha \beta \in \mathcal{H}$.

CASE 1: $\operatorname{rk}(C)<\rho$. In this case we apply (Cut) to (1) and use the fact that $\varphi \alpha \beta_{0}<\varphi \alpha \beta \in \mathcal{H}$. This gives us $\mathcal{H} \mid \frac{\varphi \alpha \beta}{\rho} \Gamma$.

CAES 2: $\operatorname{rk}(C)=\rho+\omega^{\alpha_{1}}+\ldots+\omega^{\alpha_{n}}$ with $n \geq 0, \alpha>\alpha_{1} \geq \ldots \geq \alpha_{n}$. From $\mathrm{k}(C) \subseteq \mathcal{H}$ it follows that $\operatorname{rk}(C) \in \mathcal{H}$ and thus $\alpha_{1}, \ldots, \alpha_{n} \in \mathcal{H}$.

The Reduction-Lemma applied to (1) yields (3) $\mathcal{H} \mid \frac{\varphi \alpha \beta_{0}+\varphi \alpha \beta_{0}}{\mathrm{rk}(C)} \Gamma$, and from this we obtain $\quad$ (4) $\mathcal{H} \mid \frac{\varphi \beta \beta}{\mathrm{rk}(C)} \Gamma$, since $\varphi \alpha \beta_{0}+\varphi \alpha \beta_{0}<\varphi \alpha \beta \in \mathcal{H}$. Now using $\alpha_{1}, \ldots, \alpha_{n} \in \mathcal{H}, \operatorname{rk}(C)=\rho+\omega^{\alpha_{1}}+\ldots+\omega^{\alpha_{n}}$ and $\varphi \alpha_{i}(\varphi \alpha \beta)=$ $\varphi \alpha \beta(i=1, \ldots, n)$ by $\mathrm{n}$ applications of the main I.H. we get $\mathcal{H} \mid \frac{\varphi \alpha \beta}{\rho} \Gamma$.

Corollary

$\mathcal{H}\left|\frac{\beta}{\rho+1} \Gamma \& \rho \notin \mathrm{R} \Longrightarrow \mathcal{H}\right| \frac{\omega^{\beta}}{\rho} \Gamma$

Lemma 3.17 (Boundedness)

$\mathcal{H}\left|\frac{\alpha}{\rho} \Gamma, C \& \alpha \leq \beta<\kappa \& C \in \Sigma(\kappa) \& \beta \in \mathcal{H} \Longrightarrow \mathcal{H}\right| \frac{\alpha}{\rho} \Gamma, C^{(\beta, \kappa)}$

Proof by induction on $\alpha$.

1. Suppose that $C \simeq \wedge\left(C_{\iota}\right)_{\iota \in J}$ and $\mathcal{H}[\iota] \mid \frac{\alpha_{\iota}}{\rho} \Gamma, C, C_{\iota}$ with $\alpha_{\iota}<\alpha \in \mathcal{H}$, for all $\iota \in J$. Then, since $C \in \Sigma(\kappa)$, we have $C^{(\beta, \kappa)} \simeq \wedge\left(C_{\iota}^{(\beta, \kappa)}\right)_{\iota \in J}$. By (two applications of) the I.H. we obtain $\mathcal{H}[\iota] \mid \frac{\alpha_{\iota}}{\rho} \Gamma, C^{(\beta, \kappa)}, C_{\iota}^{(\beta, \kappa)} \quad(\forall \iota \in J)$ and from this $\mathcal{H} \mid \frac{\alpha}{\rho} \Gamma, C^{(\beta, \kappa)}$ by an application of $(\Lambda)$.

2. Suppose that $C \simeq \mathrm{V}\left(C_{\iota}\right)_{\iota \in J}$ and $\mathcal{H} \mid \frac{\alpha_{0}}{\rho} \Gamma . C, C_{\iota_{0}}$ with $\iota_{0} \in J \mid \alpha$ and $\alpha_{0}<$ $\alpha \in \mathcal{H}$. Then $C^{(\beta, \kappa)} \simeq \bigvee\left(C_{\imath}^{(\beta, \kappa)}\right)_{\iota \in J^{\prime}}$ with $J^{\prime}=J$ or $J^{\prime}=J \mid \beta$. Since $\alpha \leq \beta$ and $\iota_{0} \in J \mid \alpha$, we also have $\iota_{0} \in J^{\prime} \mid \alpha$. Therefore by I.H. and $(\mathrm{V})$ we obtain $\mathcal{H} \mid \frac{\alpha}{\rho} \Gamma, C^{(\beta, \kappa)}$ as in the first case.

3. Suppose that $C \equiv \exists z \in \mathrm{L}_{\kappa} A^{(z, \kappa)}$ and $\mathcal{H} \mid \frac{\alpha_{0}}{\rho} \Gamma . C, A$ with $.1 \in \Sigma(\kappa)$ and 
$\alpha_{0}+1<\alpha \in \mathcal{H}$. Then by two applications of the I.H. we obtain

(1) $\mathcal{H} \mid \frac{\alpha_{0}}{\rho} \Gamma, C^{(\beta, \kappa)}, A^{\left(\alpha_{0}, \kappa\right)}$. We also have (2) $\mathcal{H} \mid \frac{\alpha_{0}}{\rho} \Gamma, C^{(\beta, \kappa)}, \mathrm{L}_{\alpha_{0}} \notin \mathrm{L}_{0}$.

By $(\wedge)$ from (1),(2) we get (3) $\mathcal{H} \mid \frac{\alpha_{0}+1}{\rho} \Gamma, C^{(\beta, \kappa)}, \mathrm{L}_{\alpha_{0}} \notin \mathrm{L}_{0} \wedge A^{\left(\alpha_{0}, \kappa\right)}$.

Now observe that $C^{(\beta, \kappa)} \simeq \bigvee\left(\iota \notin \mathrm{L}_{0} \wedge A^{(\iota, \kappa)}\right)_{\iota \in T_{\beta}}$, and that for $\iota:=\mathrm{L}_{\alpha_{0}}$ we have $\iota \in J \mid \alpha$ and $A^{(\iota, \kappa)} \equiv A^{\left(\alpha_{0}, \kappa\right)}$. Therefore by $(\mathrm{V})$ from (3) we obtain $\mathcal{H} \mid \frac{\alpha}{\rho} \Gamma . C^{(\beta, \kappa)}$.

4. In all other cases the assertion follows immediately from the I.H. .

\section{The Collapsing Theorem}

In this section we can no longer do with the extremely weak assumption that $\mathrm{R}$ is just a class of $\varepsilon$-numbers, but we have to assume much stronger closure properties for the elements of $R$. The most natural approach would be to define $R$ as the class of all admissible ordinals $>\omega$. But from the technical side it is much more convenient to assume that the elements of $R$ are uncountable regular cardinals. Under this assumption one can prove much more easily that the functions $\psi_{\kappa}(\kappa \in \mathrm{R})$ defined below are indeed collapsing functions, i.e. that $\psi_{\kappa} \alpha<\kappa$ holds for all $\alpha \in$ On, $\kappa \in$ R. Moreover using regular cardinals instead of admissibles does not affect the size of the ordinal $v$ which we will obtain as an upper bound for the proof theoretic ordinal of KPi.

\section{Definition 4.1}

$\Omega_{0}:=0, \Omega_{\sigma}:=\aleph_{\sigma}$ for $\sigma>0$.

We assume the existence of a weakly inaccessible cardinal, i.e. a regular fixpoint of $\sigma \mapsto \Omega_{\sigma}$, and set

$I:=\min \left\{\sigma: \sigma\right.$ regular $\left.\& \Omega_{\sigma}=\sigma\right\}$

$\mathrm{R}:=\{\sigma: \omega<\sigma \leq \mathrm{I} \& \sigma$ regular $\}=\{\mathrm{I}\} \cup\left\{\Omega_{\sigma+1}: \sigma<\mathrm{I}\right\}$

As before we use $\kappa, \pi, \tau$ to denote elements of $R$. 
Definition 4.2 (The collapsing functions $\psi_{\kappa}$ )

By transfinite recursion on $\alpha$ we define ordinals $\psi_{\kappa} \alpha$ and sets $C(\alpha, \beta) \subseteq$ On as follows

$\psi_{\kappa} \alpha:=\min \{\beta: \kappa \in C(\alpha, \beta) \& C(\alpha, \beta) \cap \kappa \subseteq \beta\}$

$C(\alpha, \beta):=\left\{\begin{array}{c}\text { the closure of } \beta \cup\{0, \mathrm{I}\} \text { under the functions } \\ +, \varphi, \sigma \mapsto \Omega_{\sigma},(\xi, \pi) \mapsto \dot{\psi}_{\pi} \xi(\xi<\alpha, \pi \in \mathrm{R})\end{array}\right.$

(Note that by I.H. $\psi_{\pi} \xi$ is already defined for all $\xi<\alpha, \pi \in$ R.)

We then set $\psi_{\kappa}:$ On $\longrightarrow$ On, $\psi_{\kappa}(\alpha):=\psi_{\kappa} \alpha$.

Definition 4.3 (The operators $\mathcal{H}_{\gamma}$ )

$\mathcal{H}_{\gamma}(X):=\bigcap\{C(\alpha, \beta): X \subseteq C(\alpha, \beta) \& \gamma<\alpha\}$

The remainder of this section is devoted to the proof of the following theorem, called Collapsing Theorem or Impredicative Cut-Elimination Theorem.

$\mathcal{H}_{0}\left|\frac{\alpha}{\mathrm{I}+1} \Gamma \& \Gamma \subseteq \Sigma\left(\Omega_{1}\right) \Longrightarrow\right| \frac{\beta}{\beta} \Gamma$ with $\beta:=\psi_{\Omega_{1}}\left(\omega^{\mathrm{I}+1+\alpha}\right)$

This theorem in combination with $3.12,3.16,3.17,3.2,1.5$ then yields the final result that $|\mathrm{KPi}| \leq \psi_{\Omega_{1}} \varepsilon_{\mathrm{I}+1}$.

The above defined functions $\psi_{\kappa}(\kappa \in \mathrm{R})$ constitute a subsystem of the system $\left(\Psi_{\kappa}: \kappa<\Lambda_{0}\right)$ introduced in (Jäger [7]) which on the other hand was obtained by extending our system $\left(\psi_{\kappa}: \kappa<\Omega_{\omega}\right)$ from (Buchholz [2]). Actually the above definition looks a little bit different from that in (Jäger [7]), but nevertheless, restricted to $\kappa \leq \mathrm{I}$, both definitions are equivalent.

Before proving the Collapsing Theorem we have to prove some basic properties of the functions $\psi_{\kappa}$ and the sets $C(\alpha, \beta)$.

Abbreviation: $\quad C_{\kappa}(\alpha):=C\left(\alpha, \dot{\psi}_{\kappa} \alpha\right)$.

Lemma 4.4

a) $\beta<\pi \Longrightarrow$ cardinality $(C(\alpha, \beta))<\pi$

b) $C(\alpha, \beta)=\cup_{\eta<\beta} C(\alpha, \eta)$, for each limit ordinal $\beta$

c) $\kappa \in C(\alpha, \kappa)$

d) $C_{\kappa}(\alpha) \cap \kappa=\psi_{\kappa} \alpha$

Proof. All statements are immediate consequences of definition 4.2. 


\section{Lemma 4.5}
a) $\psi_{\kappa} \alpha<\kappa \& \psi_{\kappa} \alpha \notin C_{\kappa}(\alpha)$
b) $\alpha_{0}<\alpha \& \alpha_{0} \in C_{\kappa}(\alpha) \Longrightarrow \psi_{\kappa} \alpha_{0}<\psi_{\kappa} \alpha$
c) $\psi_{\kappa} \alpha \notin\left\{\Omega_{\sigma}: \sigma<\Omega_{\sigma}\right\} \cup\{0\} \& \forall \xi, \eta<\psi_{\kappa} \alpha\left(\varphi \xi \eta<\psi_{\kappa} \alpha\right)$
d) $\Omega_{\sigma} \in C(\alpha, \beta) \Longrightarrow \sigma \in C(\alpha, \beta)$
e) $\omega^{\xi_{0}} \# \ldots \# \omega^{\xi_{n}} \in C(\alpha, \beta) \Longrightarrow\left\{\xi_{0}, \ldots, \xi_{n}\right\} \subseteq C(\alpha, \beta)$
f) $\kappa=\Omega_{\sigma+1} \Longrightarrow \Omega_{\sigma}<\psi_{\kappa} \alpha<\Omega_{\sigma+1}$
g) $\Omega_{\psi_{I} \alpha}=\psi_{I} \alpha$
h) $\Omega_{\sigma} \leq \gamma \leq \Omega_{\sigma+1} \& \gamma \in C(\alpha, \beta) \Longrightarrow \sigma \in C(\alpha, \beta)$
i) $\alpha_{0} \leq \alpha \Longrightarrow \psi_{\kappa} \alpha_{0} \leq \psi_{\kappa} \alpha \& C_{\kappa}\left(\alpha_{0}\right) \subseteq C_{\kappa}(\alpha)$

\section{Proof.}

a) Let $\beta_{0}:=\min \{\eta: \kappa \in C(\alpha, \eta)\}, \beta_{n+1}:=\min \left\{\eta: C\left(\alpha, \beta_{n}\right) \cap \kappa \subseteq \eta\right\}$ and $\beta:=\sup \left\{\beta_{n}: n \in \omega\right\}$. Using $\left.4.4 a\right)$ we obtain $\forall n \in \omega\left(\beta_{n} \leq \beta_{n+1}<\kappa\right)$. Hence $\beta<\kappa, \kappa \in C(\alpha, \beta)$ and $C(\alpha, \beta) \cap \kappa=\bigcup\left\{C\left(\alpha, \beta_{n}\right) \cap \kappa: n \in \omega\right\} \subseteq$ $\bigcup\left\{\beta_{n+1}: n \in \omega\right\}=\beta$. By definition of $\psi_{\kappa} \alpha$ this yields $\psi_{\kappa} \alpha \leq \beta<\kappa$. From $C_{\kappa}(\alpha) \cap \kappa=\psi_{\kappa} \alpha<\kappa$ it follows that $\psi_{\kappa} \alpha \notin C_{\kappa}(\alpha)$.

b) $\alpha_{0}<\alpha \& \alpha_{0} \in C_{\kappa}(\alpha)$ together with $\kappa \in C_{\kappa}(\alpha)$ implies $\psi_{\kappa} \alpha_{0} \in C_{\kappa}(\alpha)$. Using a) and $4.4 \mathrm{~d})$ we obtain $\psi_{\kappa} \alpha_{0} \in C_{\kappa}(\alpha) \cap \kappa=\psi_{\kappa} \alpha$.

c) Let us assume that $\dot{\psi}_{\kappa} \alpha=\Omega_{\sigma}$ with $\sigma=0$ or $\sigma<\Omega_{\sigma}$. Then by definition of $C_{\kappa}(\alpha)$ we would have $\dot{\psi}_{\kappa} \alpha \in C_{\kappa}(\alpha)$ which contradicts a). - The second part is an immediate consequence of $\psi_{\kappa} \alpha=C_{\kappa}(\alpha) \cap \kappa$ and the fact that $\kappa$ and $C_{\kappa}(\alpha)$ both are closed under $\varphi$.

d) Let us assume that $\sigma<\Omega_{\sigma}$ and $\sigma \notin C(\alpha, \beta)$. Then $\Omega_{\sigma} \notin \beta \cup\{0, I\}$ and, according to $c), \Omega_{\sigma} \neq \psi_{\pi} \xi(\forall \xi, \pi)$. Moreover we have $\forall \xi, \eta\left(\Omega_{\sigma} \in\right.$ $\left.\{\xi+\eta, \varphi \xi \eta\} \Longrightarrow \Omega_{\sigma} \in\{\xi, \eta\}\right)$. Therefore the set $C(\alpha, \beta) \backslash\left\{\Omega_{\sigma}\right\}$ contains $\beta \cup\{0, \mathrm{I}\}$ and is closed under $+, \varphi, \zeta \mapsto \Omega_{\zeta},(\xi, \pi) \mapsto \psi_{\pi} \xi(\xi<\alpha, \pi \in \mathrm{R})$. By definition of $C(\alpha, \beta)$ this implies $C(\alpha, \beta) \subseteq C(\alpha, \beta) \backslash\left\{\Omega_{\sigma}\right\}$, i.e. $\Omega_{\sigma} \notin C(\alpha, \beta)$.

e) This is proved in the same way as d), now using the fact that the ordinals $\Omega_{\sigma}$ and $\psi_{\pi} \xi$ are closed under $\varphi$.

f) From $\Omega_{\sigma+1}=\kappa \in C_{\kappa}(\alpha)$ it follows by d) and e) that $\sigma \in C_{\kappa}(\alpha)$. Hence $\Omega_{\sigma} \in C_{\kappa}(\alpha) \cap \kappa=\psi_{\kappa} \alpha$. 
g) Let $\Omega_{\sigma} \leq \psi_{\mathrm{I}} \alpha<\Omega_{\sigma+1}$. Then $\Omega_{\sigma+1}<\mathrm{I}$ and therefore $\Omega_{\sigma+1} \notin C_{\mathrm{I}}(\alpha)$, since $\Omega_{\sigma+1} \notin \psi_{\mathrm{I}} \alpha=C_{\mathrm{I}}(\alpha) \cap \mathrm{I}$. It follows that $\sigma \notin C_{\mathrm{I}}(\alpha)$ and thus $w_{\mathrm{I}} \alpha \leq \sigma \leq \Omega_{\sigma}$.

h) We assume $\sigma \notin C(\alpha, \beta)$. Then also $\Omega_{\sigma}, \Omega_{\sigma+1} \notin C(\alpha, \beta)$. Obviously $\beta \cup\{0, \mathrm{I}\} \subseteq Y:=C(\alpha, \beta) \backslash\left[\Omega_{\sigma}, \Omega_{\sigma+1}\right]$ and $Y$ is closed under $+, \varphi, \zeta \mapsto \Omega_{\zeta}$. By f) and $\mathrm{g})$ it follows that $Y$ is also closed under $(\xi, \pi) \mapsto \psi_{\pi} \xi(\xi<\alpha, \pi \in \mathrm{R})$. Hence $C(\alpha, \beta) \subseteq C(\alpha, \beta) \backslash\left[\Omega_{\sigma}, \Omega_{\sigma+1}\right]$, i.e. $C(\alpha, \beta) \cap\left[\Omega_{\sigma}, \Omega_{\sigma+1}\right]=\emptyset$.

i) By f) it follows that $\kappa \in C\left(\alpha_{0}, \psi_{\kappa} \alpha\right)$. We also have $C\left(\alpha_{0}, \psi_{\kappa} \alpha\right) \cap \kappa \subseteq$ $C\left(\alpha, \psi_{\kappa} \alpha\right) \cap \kappa=\psi_{\kappa} \alpha$. By the definition of $\psi_{\kappa} \alpha_{0}$ from $\kappa \in C\left(\alpha_{0}, \psi_{\kappa} \alpha\right)$ and $C\left(\alpha_{0}, \psi_{\kappa} \alpha\right) \cap \kappa \subseteq \psi_{\kappa} \alpha$ it follows that $\psi_{\kappa} \alpha_{0} \leq \psi_{\kappa} \alpha$.

As an immediate consequence of the above lemma we get the following which summarizes the basic closure properties of the operators $\mathcal{H}_{\gamma}$.

\section{Lemma 4.6}

a) $\mathcal{H}_{\gamma}$ is a nice operator.

b) $\mathcal{H}_{\gamma}$ is closed under $\varphi$.

c) $\xi \leq \gamma \& \xi, \pi \in \mathcal{H}_{\gamma}(X) \Longrightarrow \psi_{\pi} \xi \in \mathcal{H}_{\gamma}(X)$

d) $\Omega_{\sigma} \leq \alpha \leq \Omega_{\sigma+1} \& \alpha \in \mathcal{H}_{\gamma}(X) \Longrightarrow \Omega_{\sigma}, \Omega_{\sigma+1} \in \mathcal{H}_{\gamma}(X)$

e) $\gamma<\delta \Longrightarrow \mathcal{H}_{\gamma}(X) \subseteq \mathcal{H}_{\delta}(X)$

\section{Abbreviations}

$\overline{\mathrm{K}}:=\left\{\bar{\Omega}_{\sigma}: \sigma \leq I\right\}$ with $\bar{\Omega}_{\sigma}:=\left\{\begin{array}{cc}\Omega_{\sigma}+1 & \text { if } \Omega_{\sigma} \in \mathrm{R} \\ \Omega_{\sigma} & \text { otherwise }\end{array}\right.$ $\mathcal{A}(\Theta ; \gamma, \kappa, \mu): \Leftrightarrow \mu \in \overline{\mathrm{K}} \& \gamma, \kappa, \mu \in \mathcal{H}_{\gamma}[\Theta] \& \mathrm{k}(\Theta) \subseteq \bigcap_{\tau \geq \kappa} C_{\tau}(\gamma+1)$

\section{Lemma 4.7}

Suppose $\mathcal{A}(\Theta ; \gamma, \kappa, \mu)$. Then the following holds:

$(\mathcal{A} 1) \xi \in \mathcal{H}_{\gamma}[\Theta] \& \gamma^{\prime}=\gamma+\omega^{\mu+\xi} \Longrightarrow \gamma^{\prime} \in \mathcal{H}_{\gamma}[\Theta] \& \psi_{\kappa} \gamma^{\prime} \in \mathcal{H}_{\gamma^{\prime}}[\Theta]$

(A2) $\xi \in \mathcal{H}_{\gamma}[\Theta] \& \gamma+\omega^{\mu+\xi}<\eta \Longrightarrow \psi_{\kappa}\left(\gamma+\omega^{\mu+\xi}\right)<\psi_{\kappa} \eta$

$(\mathcal{A} 3) \quad r \leq \tau \Longrightarrow \mathcal{H}_{\gamma}[\Theta] \cap \tau \subseteq \dot{\psi}_{\tau}(\gamma+1)$

(A4) $\gamma^{\prime}<\gamma+\omega^{\mu+\alpha} \& \mu^{\prime}+\alpha^{\prime}<\mu+\alpha \Longrightarrow \gamma^{\prime}+\omega^{\mu^{\prime}+\alpha^{\prime}}<\gamma+\omega^{\mu+\alpha}$ Proof.

1. From $\xi, \gamma, \mu \in \mathcal{H}_{\gamma}[\Theta]$ by 4.6a) it follows that $\gamma^{\prime} \in \mathcal{H}_{\gamma}[\Theta]$. From $\gamma^{\prime} . \kappa \in$ $\mathcal{H}_{\gamma}[\Theta]$ \& $\gamma \leq \gamma^{\prime}$ we get $\psi_{\kappa} \gamma^{\prime} \in \mathcal{H}_{\gamma^{\prime}}[\Theta]$ by $\left.\left.4.6 \mathrm{c}\right), \mathrm{e}\right)$. 
2. Let $\gamma^{\prime}:=\gamma+\omega^{\mu+\xi}$. Then $\gamma^{\prime} \in \mathcal{H}_{\gamma}[\Theta]$ (by $(\mathcal{A} 1)$ ), and $\mathcal{H}_{\gamma}[\Theta] \subseteq C_{\kappa}(\gamma+1)$, since $\mathrm{k}(\Theta) \subseteq C_{\kappa}(\gamma+1)$. By 4.5b),i) from $\gamma^{\prime} \in C_{\kappa}(\gamma+1)$ and $\gamma<\gamma^{\prime}<\eta$ it follows that $\dot{\psi}_{\kappa} \gamma^{\prime}<\psi_{\kappa} \eta$.

3. $\mathcal{H}_{\gamma}[\Theta] \cap \tau \subseteq C_{\tau}(\gamma+1) \cap \tau=\psi_{\tau}(\gamma+1)$.

4. Obvious.

\section{Remark}

In $(\mathcal{A} 2)$ above the crucial interplay between $\mathcal{H}_{\gamma}$ and $\psi_{\kappa}$ shows up most clearly. Assuming $\mathcal{A}(\Theta ; \gamma, \kappa, \mu)$ the function $\xi \mapsto \psi_{\kappa}\left(\gamma+\omega^{\mu+\xi}\right)$ provides an order preserving map from $\mathcal{H}_{\gamma}[\Theta]$ into $\kappa$.

Theorem 4.8 (Collapsing and impredicative cutelimination) $\mathcal{A}(\Theta ; \gamma, \kappa, \mu) \quad \& \quad \Gamma \subseteq \Sigma(\kappa) \quad \& \mathcal{H}_{\gamma}[\Theta] \mid \frac{\alpha}{\mu} \Gamma \Longrightarrow$

$\Longrightarrow \mathcal{H}_{\hat{\alpha}}[\Theta] \mid \frac{\psi_{\kappa} \hat{\alpha}}{\psi_{\kappa} \hat{\alpha}} \Gamma \quad$ with $\quad \hat{\alpha}:=\gamma+\omega^{\mu+\alpha}$.

Proof by main induction on $\mu$ and subsidiary induction on $\alpha$. Abbreviation: $\mathcal{H}|\underline{\underline{\alpha}} \Gamma: \Leftrightarrow \mathcal{H}| \frac{\alpha}{\alpha} \Gamma$.

First note that from $\mathcal{A}(\Theta ; \gamma, \kappa, \mu)$ and $\alpha \in \mathcal{H}_{\gamma}[\Theta]$ by $(\mathcal{A} 1),(\mathcal{A} 2)$ we get:

(1) $\psi_{\kappa} \hat{\alpha} \in \mathcal{H}_{\hat{\alpha}}[\Theta]$

(2) $\mathcal{A}\left(\Theta^{\prime} ; \gamma, \kappa, \mu\right) \& \alpha_{0} \in \mathcal{H}_{\gamma}\left[\Theta^{\prime}\right] \& \alpha_{0}<\alpha \Longrightarrow \psi_{\kappa} \widehat{\alpha_{0}}<\psi_{\kappa} \hat{\alpha}$.

Now we distinguish cases according to the last inference of $\mathcal{H}_{\gamma}[\Theta] \mid \frac{\alpha}{\mu} \Gamma$ :

1. Suppose that $A \simeq \wedge\left(A_{\iota}\right)_{\iota \in J} \in \Gamma$ and $\mathcal{H}_{\gamma}[\Theta][\iota] \mid \frac{\alpha_{\iota}}{\mu} \Gamma$. $A_{\iota}$ with $\alpha_{\iota}<\alpha$ for all $\iota \in J$. Since $A \in \Gamma \subseteq \Sigma(\kappa)$, there is a $\beta \in \mathrm{k}(A) \cap \kappa$ such that $\forall \iota \in J(|\iota| \leq 3)$. Since $\mathrm{k}(A) \subseteq \mathcal{H}_{\gamma}[\Theta]$, by $(\mathcal{A} 3)$ it follows that $\forall \tau \geq \kappa\left(\beta<\psi_{\tau}(\gamma+1)\right)$ and thus $\forall \iota \in J \forall \tau \geq \kappa\left(\mathrm{k}(\iota) \subseteq C_{\tau}(\gamma+1)\right)$. Hence $\mathcal{A}(\Theta, \iota ; \gamma, \kappa, \mu)$ and therefore (by S.I.H.) $\mathcal{H}_{\hat{\alpha}}[\Theta][\iota] \mid \frac{\psi_{\kappa} \widehat{\alpha_{\iota}}}{\cdot} \Gamma, A_{\iota}$ for all $\iota \in J$. From this we obtain $\mathcal{H}_{\hat{\alpha}}[\Theta] \mid \frac{\psi_{\kappa} \hat{\alpha}}{.} \Gamma$ by $(\Lambda)$ and $(1),(2)$.

2. Suppose that $\bigvee\left(A_{\iota}\right)_{\iota \in J} \in \Gamma$ and $\mathcal{H}_{\gamma}[\Theta] \mid \frac{\alpha_{0}}{\mu} \Gamma$. $A_{\iota 0}$ with $\iota_{0} \in J$ and $\alpha_{0}<\alpha$. By S.I.H. we obtain $\mathcal{H}_{\hat{\alpha_{0}}}[\Theta] \mid \frac{\psi_{\kappa} \widehat{\alpha_{0}}}{\cdot} \Gamma, A_{\iota_{0}}$ and then $\mathcal{H}_{\hat{\alpha}}[\Theta] \mid \frac{\psi_{\kappa} \hat{\alpha}}{\cdot} \Gamma$ using $(V)$, (1), (2) and $k\left(\iota_{0}\right) \subseteq k\left(A_{\iota_{0}}\right) \cap \kappa \subseteq \mathcal{H}_{\gamma}[\Theta] \cap \kappa \subseteq \psi_{\kappa}(\gamma+1) \subseteq \dot{\psi}_{\kappa} \hat{\alpha}$.

3. If the last inierence was an instance of (Ref) then the assertion follows immediately from (1), (2), and the S.I.H. . 
Before treating (Cut) we prove the following proposition.

() Assume $\gamma \leq \gamma^{\prime}<\hat{\alpha} \& \gamma^{\prime} \in \mathcal{H}_{\gamma^{\prime}}[\Theta] \& \operatorname{rk}\left(C^{\prime}\right), \beta<\pi \leq \mu$ and $\mathcal{H}_{\gamma^{\prime}}[\Theta] \mid \frac{\beta}{.} \Gamma,(\neg) C^{\prime} . \quad$ Then $\mathcal{H}_{\hat{\alpha}}[\Theta] \mid \frac{\psi_{\kappa} \hat{\alpha}}{\cdot} \Gamma$.

Proof. Let $\rho:=\max \left\{\operatorname{rk}\left(C^{\prime}\right), \beta\right\}+1$ and $\sigma \in$ On such that $\Omega_{\sigma}<\rho<\Omega_{\sigma+1}$. Then for $\mu^{\prime}:=\bar{\Omega}_{\sigma}$ we have $\left[\mu^{\prime}, \mu^{\prime}+\omega^{\rho}\right] \cap \mathrm{R}=\emptyset$ and $\mu^{\prime} \in \mathcal{H}_{\gamma^{\prime}}[\Theta]$ (since $\rho \in$ $\left.\mathcal{H}_{\gamma^{\prime}}[\Theta]\right)$. By (Cut) we obtain $\mathcal{H}_{\gamma^{\prime}}[\Theta] \mid \frac{\beta+1}{\mu^{\prime}+\omega^{\rho}} \Gamma$, and then by the Predicative Cut-Elimination Theorem $\mathcal{H}_{\gamma^{\prime}}[\Theta] \mid \frac{\alpha^{\prime}}{\mu^{\prime}} \Gamma$ with $\alpha^{\prime}:=\varphi \rho(\beta+1)$. From $\mu^{\prime} \in \mathcal{H}_{\gamma^{\prime}}[\Theta]$ together with $\gamma \leq \gamma^{\prime} \in \mathcal{H}_{\gamma^{\prime}}[\Theta]$ and $\mathcal{A}(\Theta ; \gamma, \kappa, \mu)$ we obtain $\mathcal{A}\left(\Theta ; \gamma^{\prime}, \kappa, \mu^{\prime}\right)$. Since $\mu^{\prime}<\mu$, we can now apply the M.I.H. and obtain $\mathcal{H}_{\alpha} \cdot[\Theta] \mid \frac{\psi_{\kappa} \alpha^{*}}{.} \Gamma$ with $\alpha^{*}:=\gamma^{\prime}+\omega^{\mu^{\prime}+\alpha^{\prime}}$. Since $\mu^{\prime}+\alpha^{\prime}<\pi \leq \mu \quad \& \quad \gamma^{\prime}<\hat{\alpha}$, we have $\alpha^{*}<\hat{\alpha}$ and $\psi_{\kappa} \alpha^{*} \leq \psi_{\kappa} \hat{\alpha}$. Hence $\mathcal{H}_{\hat{\alpha}}[\Theta] \mid \frac{\psi_{\kappa} \hat{\alpha}}{\cdot} \Gamma$.

4. Suppose that $\mathcal{H}_{\gamma}[\Theta] \mid \frac{\alpha_{0}}{\mu} \Gamma,(\neg) C$ with $\alpha_{0}<\alpha$ and $\operatorname{rk}(C)<\mu$.

4.1. $\operatorname{rk}(C)<\kappa$.

Since $\mathrm{k}(C) \subseteq \mathcal{H}_{\gamma}[\Theta]$, we then have $\operatorname{rk}(C) \in \mathcal{H}_{\gamma}[\Theta] \cap \kappa \subseteq \psi_{\kappa}(\gamma+1) \subseteq \dot{\psi}_{\kappa} \hat{\alpha}$, and the assertion follows immediately from the S.I.H.

4.2. $\kappa \leq \mathrm{rk}(C) \notin \mathrm{R}$.

Let $\pi:=\operatorname{rk}(C)^{\mathrm{R}}$. Then $\kappa \leq \operatorname{rk}(C)<\pi \leq \mu \quad \& \quad \pi \in \mathcal{H}_{\gamma}[\Theta]$. Hence $\mathcal{A}(\Theta ; \gamma, \pi, \mu)$. Since $C, \neg C \in \Sigma(\pi)$, the S.I.H. yields $\mathcal{H}_{\widehat{\alpha_{0}}}[\Theta] \mid \frac{\psi_{\pi} \widehat{\alpha_{0}}}{.} \Gamma,(\neg) C$. From $\gamma, \alpha_{0}, \mu \in \mathcal{H}_{\gamma}[\Theta] \& \gamma<\widehat{\alpha_{0}}$ we obtain $\widehat{\alpha_{0}} \in \mathcal{H}_{\widehat{\alpha_{0}}}[\Theta]$. Now the assertion follows by $(\square)$.

4.3. $\kappa \leq \operatorname{rk}(C)=\pi$.

W.l.o.g. we have $C \equiv \exists x \in \mathrm{L}_{\pi} A(x) \in \Sigma(\pi)$. We also have $\kappa \leq \pi \in \mathcal{H}_{\gamma}[\Theta]$ and thus $\mathcal{A}(\Theta ; \gamma, \pi, \mu)$. Now the S.I.H. gives us $\mathcal{H}_{\widehat{\alpha_{0}}}[\Theta] \mid \frac{\beta}{.} \Gamma, C$ with $\beta:=\psi_{\pi} \widehat{\alpha_{0}}$. By the Boundedness-Lemma from this we get

(3) $\mathcal{H}_{\hat{\alpha_{0}}}[\Theta] \mid \frac{\beta}{\cdot} \Gamma, \exists x \in \mathrm{L}_{\beta} A(x)$.

Now we apply Lemma 3.9c) to the premise $\mathcal{H}_{\gamma}[\Theta] \mid \frac{\alpha_{0}}{\mu} \Gamma . \neg C$.

Since $\neg C \equiv \forall x \in \mathrm{L}_{\pi} \neg A(x)$ and $\beta \in \mathcal{H}_{\widehat{\alpha_{0}}}[\Theta] \cap \pi$. this gives us

(4) $\mathcal{H}_{\widehat{\alpha_{0}}}[\Theta] \mid \frac{\alpha_{0}}{\mu} \Gamma . \forall x \in \mathrm{L}_{\beta} \neg A(x)$. 
From $\mathcal{A}(\Theta ; \gamma, \pi, \mu)$ and $\gamma<\widehat{\alpha_{0}} \in \mathcal{H}_{\gamma}[\Theta]$ we get $\mathcal{A}\left(\Theta ; \widehat{\alpha_{0}}, \pi, \mu\right)$. Therefore we can apply the S.I.H. to (4) and obtain

(5) $\mathcal{H}_{\gamma^{\prime}}[\Theta] \mid \frac{\psi_{\pi} \gamma^{\prime}}{\cdot} \Gamma, \forall x \in \mathrm{L}_{\beta} \neg A(x)$ with $\gamma^{\prime}:=\widehat{\alpha_{0}}+\omega^{\mu+\alpha_{0}}$.

For $C^{\prime}: \equiv \exists x \in \mathrm{L}_{\beta} A(x)$ we now have

$\gamma \leq \gamma^{\prime}<\hat{\alpha} \& \gamma^{\prime} \in \mathcal{H}_{\gamma^{\prime}}[\Theta] \& \operatorname{rk}\left(C^{\prime}\right), \psi_{\pi} \gamma^{\prime}<\pi \leq \mu \& \mathcal{H}_{\gamma^{\prime}}[\Theta] \mid \frac{\dot{\psi}_{\pi} \gamma^{\prime}}{\cdot} \Gamma,(\neg) C^{\prime}$.

Hence by $(\square)$ we obtain $\mathcal{H}_{\hat{\alpha}}[\Theta] \mid \frac{\psi_{\kappa} \hat{\alpha}}{.} \Gamma$.

\section{Corollary}

$\mathcal{H}_{0}\left|\frac{\alpha}{\mathrm{I}+1} \Gamma \& \Gamma \subseteq \Sigma\left(\Omega_{1}\right) \Longrightarrow\right| \frac{\beta}{\beta} \Gamma$ with $\beta:=\psi_{\Omega_{1}}\left(\omega^{\mathrm{I}+1+\alpha}\right)$.

\section{Theorem 4.9 (MAIN THEOREM)}

Let $v:=\psi_{\Omega_{1}}\left(\varepsilon_{\mathrm{I}+1}\right)$. Then for each $\Sigma_{1}$-sentence $\phi$ of $\mathcal{L}$ we have:

$$
\mathrm{KPi} \vdash \forall x\left(\operatorname{Ad}(x) \rightarrow \phi^{x}\right) \Longrightarrow L_{v} \vDash \phi .
$$

\section{Proof.}

Suppose that KPit $\forall x\left(A d(x) \rightarrow \phi^{x}\right)$. Then we get successively

(1) $\mathcal{H}_{0} \mid \frac{\omega^{\mathrm{I}+m}}{\mathrm{I}+m} \forall x \in \mathrm{L}_{\mathrm{I}}\left(A d(x) \rightarrow \phi^{x}\right)$, for some $m \in \mathbb{N} \quad$ [by $3.12,4.6 \mathrm{a}, \mathrm{d}$ ]

(2) $\mathcal{H}_{0} \mid \frac{\omega^{\mathrm{I}+m}}{\mathrm{I}+m} \mathrm{~L}_{\Omega_{1}} \notin \mathrm{L}_{\mathrm{I}}, \neg A d\left(\mathrm{~L}_{\Omega_{1}}\right), \phi^{\Omega_{1}} \quad$ [by 3.9b, 3.13. $\left.\Omega_{1} \in \mathcal{H}_{0}\right]$

(3) $\mathcal{H}_{0} \mid \frac{\omega^{\mathrm{I}+m}}{\mathrm{I}+m} \neg A d\left(\mathrm{~L}_{\Omega_{1}}\right), \phi^{\Omega_{1}} \quad$ [ since $\left.\mathrm{L}_{\Omega_{1}} \notin \mathrm{L}_{I} \simeq \mathrm{V}\left(A_{\iota}\right)_{\iota \in \emptyset}\right]$

(4) $\mathcal{H}_{0} \mid \frac{\omega^{I+m}+1}{I+m} \phi^{\Omega_{1}} \quad[$ by $2.5 \mathrm{~h}, 3.10$. (Cut)]

(5) $\mathcal{H}_{0} \mid \frac{\alpha}{1+1} \phi^{\Omega_{1}}$ with $\alpha<\varepsilon_{\mathrm{I}+1} \quad$ [by 3.16 (Corollary)]

(6) $\mid \frac{\beta}{\beta} \phi^{\Omega_{1}}$ with $3:=\psi_{\Omega_{1}}\left(\omega^{\mathrm{I}+1+\alpha}\right) \quad$ [by 4.8 (Corollary)]

(7) $1 \frac{\beta}{\beta} \phi^{\beta} \quad[$ by 3.17$]$

(8) $L_{v}=\varnothing \quad[$ by $3.2 .1 .5,4.5 \mathrm{i}]$

As shown in the introduction 4.9 together with [S](Th.4.6) yields Corollary. $\quad|\mathrm{KPi}| \leq \psi_{\Omega_{1}}\left(\varepsilon_{\mathrm{I}+1}\right)$. 


\section{References}

[1] Arai,T.: Proof theory for reflecting ordinals II: recursively Mahlo ordinals. Handwritten notes (1989).

[2] Buchholz,W.: A new system of proof-theoretic ordinal functions. Annals of Pure and Applied Logic 32 (1986), 195-207.

[3] Buchholz,W.: A note on the ordinal analysis of KPM. In: J.Väänänen (ed.) Proceedings Logic Colloquium '90. To appear.

[4] Jäger,G.: Die konstruktible Hierarchie als Hilfsmittel zur beweistheoretischen Untersuchung von Teilsystemen der Analysis. Dissertation, München 1979.

[5] Jäger,G.: Zur Beweistheorie der Kripke-Platek-Mengenlehre über den natürlichen Zahlen. Arch. math. Logik 22 (1982), 121-139.

[6] Jäger,G.: Iterating admissibility in proof theory. In J.Stern (ed.): Proceedings of the Herbrand Logic Colloquium '81. North-Holland (1982), 137-146.

[7] Jäger,G.: $\rho$-inaccessible ordinals, collapsing functions and a recursive notation system. Arch. math. Logik 24 (1984), 49-62.

[8] Jäger,G.: Theories for admissible sets: a unifying approach to proof theory. Bibliopolis, Napoli 1986.

[9] Jäger,G. and Pohlers, W.: Eine beweistheoretische Untersuchung von $\left(\Delta_{2}^{1}-\mathrm{CA}\right)+(\mathrm{BI})$ und verwandter Systeme. Sitzungsberichte der Bayer. Akademie der Wissenschaften, Mathematisch-Naturwissenschaftliche Klasse (1982).

[10] Pohlers, W.: Cut elimination for impredicative infinitary systems, part I: Ordinal analysis of $\mathrm{ID}_{1}$. Arch. math. Logik 21 (1981), 69-87.

[11] Pohlers, W.: Proof-theoretical analysis of $\mathrm{ID}_{\nu}$ by the method of local predicativity. In: W.Buchholz,S.Feferman,W.Pohlers, W.Sieg:Iterated Inductive Definitions and Subsystems of Analysis: Recent Proof-Theoretical Studies. Springer Lecture Notes in Mathematics 897 (1981) 
[12] Pohlers, W.: Cut elimination for impredicative infinitary systems,part II: Ordinal analysis for iterated inductive definitions. Arch. math. Logik 22 (1982), 113-129.

[13] Pohlers,W.: Contributions of the Schütte school in Munich to proof theory. In: G.Takeuti: Proof Theory 2nd edition. North-Holland (1987)

[14] Pohlers, W.: Ordinal functions and notations based on a hierarchy of inaccessible cardinals. Annals of Pure and Applied Logic 33 (1987).

[15] Pohlers, W.: Proof theory and ordinal analysis. Arch. math. Logic 30 (1991), 311-376.

[16] Pohlers,W.: A Short Course in Ordinal Analysis. This Volume.

[17] Rathjen,M.: Untersuchungen zu Teilsystemen der Zahlentheorie zweiter Stufe und der Mengenlehre mit einer zwischen $\Delta_{2}^{1}$-CA und $\Delta_{2}^{1}-\mathrm{CA}+\mathrm{BI}$ liegenden Beweisstärke. Dissertation Münster 1988.

[18] Rathjen,M.: Ordinal Notations Based on a Weakly Mahlo cardinal. Arch. math. Logic 29 (1990), 249-263.

[19] Rathjen,M.: Proof-Theoretic Analysis of KPM. Arch. math. Logic 30 (1991), 377-403.

[20] Tait,W.W.: Normal derivability in classical logic. In: J.Barwise (ed.) The Syntax and Semantics of Infinitary Languages. Springer Lecture Notes in Mathematics 72 (1968), 204-236

[21] Tait,W.W.: Applications of the cut elimination theorem to some subsystems of classical analysis. In: Intuitionism and Proof Theory. Proceedings of the summer conference at Buffalo, N.Y. 1968 A.Kino,J.Myhill, R.E.Vesley (eds.) North-Holland 1970, 475-488.

Mathematisches Institut der

Universität München

Theresienstr. 39

D-8000 München 2

Germany 